\title{
Methods and Models for Electric Load Forecasting: A Comprehensive Review
}

\author{
Mahmoud A. HAMMAD¹, Borut J EREB2², Bojan Rosi², Dejan DRAGAN2* \\ ${ }^{1}$ Arab Aca demy for Science, Technology and Maritime Transport, Alexandria, Egypt \\ 2 University of Maribor/Fa culty of Logistics, Celje, Slovenia
}

[Corresponding Author indic ated by an asterisk *]

\begin{abstract}
Elec tric load forecasting (EUF) is a vital process in the planning of the electric ity industry and plays a crucial role in electric capacity scheduling and power systems management and, therefore, it has attracted increasing academic interest Hence, the accuracy of electric load forecasting has greatimportance forenergy generating capacity scheduling and power system management. This paper presents a review of forecasting methods and models forelectric ity load. About 45 academic papers have been used for the comparison based on specified criteria such as time frame, inputs, outputs, the scale of the project, and value. The review reveals that despite the relative simplicity of all reviewed models, the regression analysis is still widely used and efficient for long-term forecasting. As for short-tem predictions, machine leaming orartificial intelligence-based models such as Artificial Neural Networks (ANN), Support Vector Machines (SVM), and Fuzzy logic are favored.
\end{abstract}

Key words - Electric load forecasting; Modeling electric ity loads; Methods and models of forecasting.

\section{INTRODUCTION}

Electric is a clean and efficient source of energy, which plays an irreplaceable role in our daily life [1]]. The signific ance of electric ity has been increasing drastic ally recently [2], and therefore it has become an essential subject in research [3] . Besides, electric power is more suitable and effic ient for the requirement of environment-friendly society compared with other traditional sourc es of energy such as natural gas, coal, and oil [1]. However, electric ity as a product has different cha racteristic $S$ compared to material products since it cannot be stored in bulk as it should be generated as soon as it is demanded $[2, \underline{4}]$. Also, the demand pattem of electric ity is complex due to the deregulation of electric ity markets such as electric ity oversupply and shortage, which could lead to inaccurate forecasting and causes signific ant financial loss [ $\underline{3}, 4]$. Moreover, the global electric ity demand is expected to multiply with inc reasing population and living standards improvement. Also, economies expand as well a susing high-power electrical a ppliances and developing technology such as sma rt grids, electric cars, and renewable energy production. All these factors make it diffic ult to manage the power system $[\underline{3}, \underline{5}, \underline{6}]$. Hence, it is nec essary to predict the needs/loa ds of electric ity in a dvance and before deciding on the generation of it [7]].

Electric load (EL)/demand forecasting is a vital process in the planning of the electric ity industry [4] and plays a crucial role in the operation of electric power systems [3] . The electric power load forecast is highly related to the economy's development, and it is also related to national security and the daily operation of soc iety []] . Therefore, the accuracy of electric load forecasting has great importance for energy generating capacity scheduling and power system management [1]], as these accurate forecasts lead to substantial savings in operating and maintenance costs, and correct decisions forfuture development [4]. Furthemore, electric power load forecasting represents the initial step in developing future generation, transmission, and distribution facilities [8]. However, the accuracy of electric load forecasting (ELF) cannot often fulfill our desired result because it is 
influenced by various uncerta in and uncontrollable factors such as economic development, human social a ctivities, country polic ies, and climate change [9].

So far, there is no precise standard for classifying the range of load forecasts [3]]. However, some authors have divided load forec asting in terms of the prediction duration into three categories $[\underline{4}, \underline{6}$, $10,11]$ : short-tem forecasts, medium-term forec asts, and long-term forecasts. Other researchers go for classifying load forecasting into four groups [3, 12-14]: long-term forecasts, mid-term forecasts, short-term forecasts, and very short-term forecasts, as follows:

- Long-term load forecasting (LTIF): is for more than one year to 20 yea rs a head. This type of forec ast is fundamental for strategic planning, construction of new generations, and develops the power supply and delivery system (generation units, tra nsmission system, and distribution system).

- Medium-term load forecasting (MTL): is usually for a week up to a year, which is used for maintenance scheduling and planning fuel purchases as well as energy trading and revenue assessment for the utilities.

- Short-term load forecasting (STIF): is for intervals ranging from one hour to a week, it is very important for day-to-day operations of a utility, schedule the generation and transmission of electric ity.

- Ultra/very short-term load forecasting (VSTIF): ranges from a few minutes to an hour ahead and is used for real-time control.

Although numerous forecasting methodsand models were developed to compute an accurate load forecasting, finding a $n$ a ppropriate forecasting model for a spec ific electric ity network is not a $n$ easy task, and none of them can be generalized for all demand pattems $[\underline{4}, \underline{9}, \underline{15}]$. Ba sed on $[\underline{1}]$, electric load forecasting modelscan be divided into two types:

- multi-factor forecasting methods, a nd

- time series forecasting methods.

The multi-factor/cross-sectional forecasting method focuses on the search of the causal relationships between different influencing factors and forecasting values. On the other hand, the time series forecasting method is depending more on the historical series. Accordingly, lots of researchers tum to utilize the time series forecasting method to forecast electric load to avoid the complicated and non-objective factors that might effect on establishing an accurate forecasting model using a multi-factor forecasting method. Thus, the time series forecasting method is much easier and quicker. The most frequently and widely used time series forecasting models can be divided into three subcategories [1]:

\section{statistical models,}

mac hine leaming models, and

\section{hybrid models.}

The main contribution of this paper is believed to be enriching the existing literature about electric load forecasting, with the main emphasis to complement the explanation given in already published review papersfrom the field. In thiscontext, there have been three most important papers detected about a comprehensive review regarding the methods, models, and different methodologies about the electric load forecasting [16-18]. Four review papers disc uss about the computational intelligence, data mining, and machine leaming approaches in the ELF field [19-22], while the two papers address a long-term ELF comprehensively $[23,24]$. One paper is entirely dedic ated to the short-term ELF only [25], while the work of Weron [26] focuses its attention to the statistic al a pproaches for modeling and forecasting electric ity loads and pric es. Finally, the review and analysis of machine leaming and regression models related to commercial buildings are presented in the paper of Yild iz and his colleagues [27].

The rest of the paperisorganized asfollows. In the next section, the most frequently used methods and models of load forecasting in the literature are briefly explained. Section III applies the methodology used in this work. In Section IV, the research findings and results are discussed and a nalyzed. Finally, in Section V, the conclusions of the paper are given. 


\section{THE UTERATURE REVIEW}

In this section, the most widely used methodsand models in the a rea of electric load forecasting are briefly discussed by reviewing the relevant previous works. According to Feinberg [28], the majority of forecasting methods for the ELF forecasting are related with the artificial intelligence a lgorithms and statistic al a pproaches. In these two spheres, the regression models, fuzzy logic, neural networks, and expert systems are particularly important. Moreover, for the medium-term and longterm forecasting, an econometric approach and so-called "end-use" approach are also the prevailing ones. On the other side, for the short-term forecasting, the following approaches are significant: neural networks, various time series and regression models, statistical leaming approaches, so-called "similar day a pproach", fuzzy logic models, and expert systems.

\section{A. Basic Forecasting/Prediction Methods}

According to $[13, \underline{29}, \underline{30}]$, the basic forecasting methods are classified into two basic types: qualitative and quantitative methods, and selecting the appropriate type depends mainly on the data available. In qualitative/subjective forecasting methods, the future load is predicted subjectively based on using the opinions of experts; however, they are not purely guesswork, but they are developed structured approaches for obtaining good forecasts without using historical data. Hence, such methods are useful and implemented when historic al data a re not a vailable or scarce. These methods include: subjective c urve fitting, the Delphi method, and technological comparisons. On the otherhand, the qua ntita tive/objec tive forec asting methodsa re ba sed on ma thematic al and statistical formulations. They are applied when the data are available, but two conditions must be satisfied: numerical info mation a bout the past is a va ila ble, and it is rea sona ble to assume that some aspects of the past pattems will continue in the future. The qua ntitative forecasting methods involve a wide range of methods, and each method hasits own properties, accuracies, and costs that must be considered when choosing a specific method within specific disciplines for specific purposes. Quantitative methods include, among the others: regression analysis, decomposition methods, exponential smoothing, and the Box-J enkins methodology. Most quantitative prediction problems demand either the time series data collected at regular periods over time or cross-sectional data, which a re collected at a single point in time. In order to summanize the structure/types of forecasting methods, one of the ways of classific ation can be composed as shown in Fig. 1 (a) $[\underline{13}, \underline{29}, \underline{30}]$. Regarding the basic classific ation of the forecasting models (disc ussed in the next section), one of the possible ways is shown in Fig. 1 (b). An illustration of the more precise classification of the forecasting models will follow later in the text (c.f. Fig. 4).

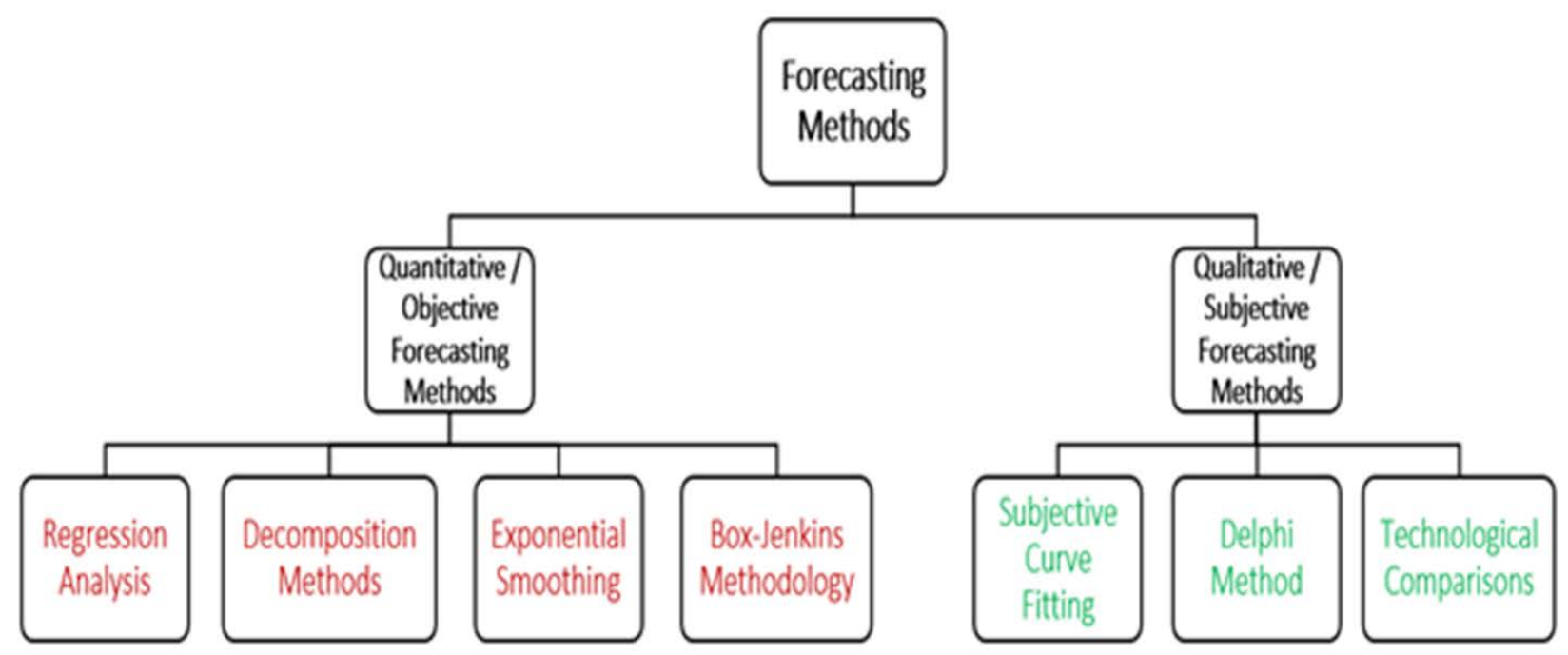


a) One of the possible classific ations of the major types of basic forecasting methods.

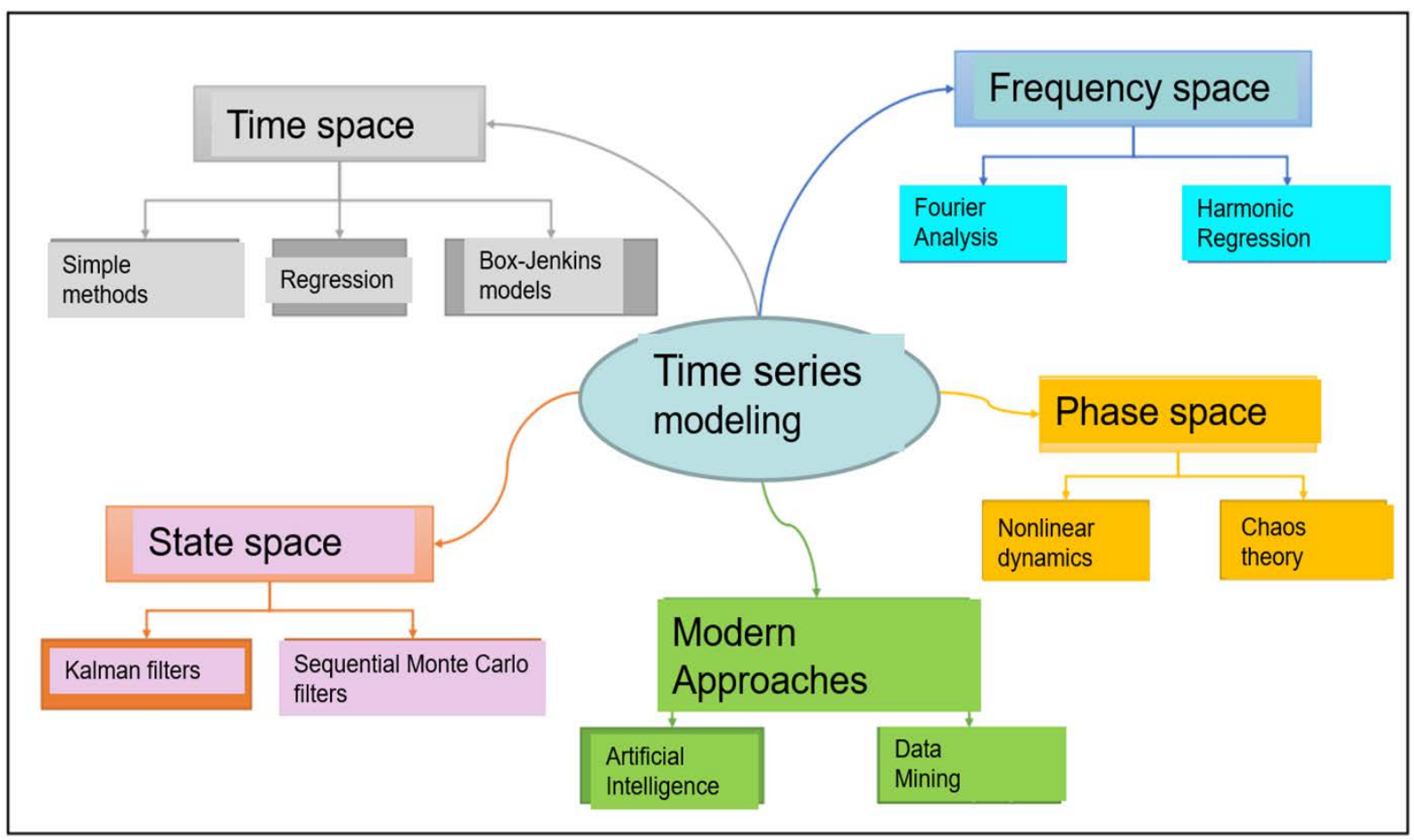

b) One of the possible basic classific ations of the forec asting models.

Figure 1: Classific ation of the majortypes of basic forecasting methodsand forecasting models

\section{B. Forecasting Models}

The early electrical load forecasting models were almost entirely limited to traditional statistical methods, but with the progress of modem science, load forecasting technologies have been considerably developed. Recently, forecasting models based on machine leaming theories are becoming more and more popular in the power load forecasting [14]. This section defines and describes the most commonly used load forecasting models, whether traditional or modem intelligent models (c.f. figure 1b) ). At this place, it is worth to mention that Fig. 1b) includes only some of the most commonly used clusters of the forecasting models. In the sequel, the discussion about the forecasting models is going to be directed in the following two directions:

\section{- 1. Statistical models.}

\section{- 2. Modem models based on machine leaming, data mining, and artificial intelligence approaches.}

\section{Statistical Models}

The statistical model is a mathematical model that embodies a set of statistical assumptions conceming the generation of sample data. A statistical model represents the data-generating process with a considerably idealized form. The statistical model is usually specified as a mathematical relationship between one or more random variablesand othernon-random variables. Several statistical models have been developed for making predictions and forecasting, according to some criteria of optimal fit [31]. In the sequel, the following models are going to be briefly disc ussed:

- Box-J enkins basic models (AR, MA, ARMA, ARIMA, ARMAX, and ARIMAX) [32-34].

- Kalman Filtering Algorithms in the State space [35-40]. 


\section{- Grey models [41-50].}

\section{- Exponential Smoothing (e.g., see [51] for an EIF, [52-54] for a general explanation).}

\section{Autoregressive (AR) Model}

The main idea of autoregressive models is that the current value of the series, $y_{t}$, can be expressed as a linear combination of previous/past loads, then the AutoRegressive (AR) model can be used to forec ast future load values. A $p$ th-order a utoregressive, $\operatorname{AR}(p)$, model is defined as:

$$
y_{t}-\sum_{i=1}^{p} \emptyset_{i} y_{t-\mathrm{i}}=\varepsilon_{t},
$$

where: $\emptyset_{1}, \emptyset_{2}, \ldots, \emptyset_{p}$ are the unknown AR coeffic ients, while $\varepsilon_{t}$ is random white noise. The order of the model tells how many lagged past values are involved. Thus, the AR model can predict future behavior based on past behaviors. It is used to forecast when there is some correlation between the current values of $y_{t}$ in a time series and its past values, where $y_{t}$ is also disturbed with the random noise $\varepsilon_{t}$. Autoregressive models have been used for decades in many fields, such as economics, elec tric load forec asting, and digital signal proc essing $[\underline{15}, \underline{29}, \underline{55}]$.

\section{Moving Average (MA) Model}

The moving average model that mimics the behavior of the moving average process, is a linear regression model that regresses the current valuesaga inst the white noise of one ormore past values. l.e. Moving a verage model can also be treated as a model in which the time series is regarded as a moving a verage (unevenly weighted) of a random shock series $\varepsilon_{t}$. Thus, the moving a verage model of order $q$ "MA $(q)$ " is given by:

$$
y_{t}=\varepsilon_{t}+\sum_{i=1}^{q} \theta_{i} \varepsilon_{t-\mathrm{i}},
$$

The noise series can be approximate by the forecast emrors or model's residuals when the load observations become available. There exists a "duality", i.e., invertibility principle between the MA process and the $A R(\infty)$ process, that is, the moving average model can be rewritten (inverted) into an a utoregressive form (of infinite order). However, thisc an only be done if the MA parameters follow certain conditions, that is if the model is invertible. Otherwise, the Box-J enkins requirements about stationarity, invertibility, and stability of the model will be violated. [포, $\underline{29}, \underline{30}, \underline{55}]$.

\section{Autoregressive Moving Average (ARMA) Model}

The Autoregressive Moving Average has been introduced in 1970 by George Boxand G wilym J enkins $[15, \underline{32}]$. The $\operatorname{ARMA}(p, q)$ models represent a combination of an a utoregressive models $\mathrm{AR}(p)$ and a moving average models MA $(q)$. In the ARMA models, the current value $y_{t}$ is expressed linearly in terms of its past values and in tems of current and previous values of the noise. Mathematically an $\operatorname{ARMA}(p, q)$ model is written as $[\underline{29}, \underline{55}, \underline{56}]$ :

$$
y_{t}-\sum_{i=1}^{p} \emptyset_{i} y_{t-\mathrm{i}}=\varepsilon_{t}+\sum_{i=1}^{q} \theta_{i} \varepsilon_{t-\mathrm{i}},
$$

ARMA models have been a popularchoice and extensively applied to load forecasting researches due to their relative simp lic ity and effectiveness [ $[\underline{5}, \underline{57}]$. 


\section{Autoregressive Integrated Moving Average (ARIMA) Model}

The AR, MA, or ARMA models, discussed above, can only be used for stationary time series data. Although in practice, many time series like those related to business and socio-economic possess a non-stationary behavior. Thus from the application perspective, the ARMA model is inadequate to describe the non-stationary time series appropriately. Therefore, the ARIMA models were proposed by Boxand J enkins in 1976 with a purpose to include the case of non-stationarity as well. The ARIMA Box-J enkins models have three types of parameters: the autoregressive parameters $\left(\emptyset_{1}, \emptyset_{2}, \ldots, \emptyset_{p}\right)$, the moving average parameters $\left(\theta_{1}, \ldots, \theta_{q}\right)$, and the number of differencing $d$ conducted to (1- $B$ ), where $B$ represents a lag operator. The mathematic al formulation of the $\operatorname{ARIMA}(p, d, q)$ model using the lag polynomia is is given below:

$$
\begin{gathered}
\emptyset(B) \cdot \nabla^{d} \cdot y_{t}=\theta(B) \cdot \varepsilon_{t}, \text { i. e., } \\
{\left[1-\sum_{i=1}^{p} \emptyset_{i} B^{i}\right] \cdot[1-B]^{d} \cdot y_{t}=\left[1+\sum_{j=1}^{q} \theta_{j} B^{j}\right] \cdot \varepsilon_{t}}
\end{gathered}
$$

where $p$ represents the order of the autoregressive, $q$ represents the moving a verage terms, and $d$ is the number of differences to make the original time series stationary. The general ARIMA $(p, d, q)$ model is a non-seasonal model, and therefore the seasonal model for it is considered as an extension of the general one which can be written as ARIMA $(p, \mathrm{~d}, q) \times(P, D, Q)_{s}$, where $s$ refers to the number of periods per sea son and $\mathrm{P}, \mathrm{D}$ and $\mathrm{Q}$ are the seasonal equivalents of $p$, d, and $q$. Hence, the sea sonal variants of ARIMA model are known as (SARIMA) models. Another useful generalization of ARIMA models is the Autoregressive Fractionally Integrated Moving Average (ARFIMA) model, whic $h$ allows non-integer values of the differencing parameter $d$. The ARFIMA has useful applic ations in modeling time series with a long memory. Accordingly, as detected in the literature, the ARIMA models and their variants have a chieved considera ble success for elec tric load forecasting $[\underline{4}, \underline{29}, \underline{55}, \underline{56}, \underline{58}, \underline{59}]$.

\section{ARMAX and ARIMAX Models}

The ARMA and ARIMA models use only the time and load as input parameters, besides the random noise that disturbs the whole process. Because loads depend on the weather and time of the day, exogenous varia bles sometimescan be included to give the ARMAX and ARIMAX models [29]. In the ARMAX (a uto regressive moving a vera ge with exogenous inputs) model, the current value of the time series $y_{t}$ is expressed linearly in terms of its past values, in terms of c urrent and previous values of the noise, and additionally, in terms of present and past values of the exogenous variable(s). The $\operatorname{ARMAX}\left(p, q, r_{1}, \ldots \ldots, r_{k}\right)$ can be compactly written as:

$$
\begin{aligned}
& \emptyset(B) \cdot y_{t}=\theta(\mathrm{B}) \cdot \varepsilon_{t}+\sum_{i=1}^{k}\left(\psi_{0}^{i}+\psi_{1}^{i} \cdot \mathrm{B}+\cdots+\psi_{r i}^{i} \cdot B^{r i}\right) \cdot v_{t}^{i}, \\
& \varnothing(B) \cdot y_{t}=\theta(\mathrm{B}) \cdot \varepsilon_{t}+\sum_{i=1}^{k} \psi^{i}(B) \cdot v_{t}^{i}
\end{aligned}
$$

where the $r_{\mathrm{i}}$ 's are the orders of the exogenous factors (variables) $v_{t}^{i}$ and $\psi^{i}(\mathrm{~B}$ ) is a shorthand notation for $\psi^{i}(\mathrm{~B})=\left(\psi_{0}^{i}+\psi_{1}^{i}\right.$. B $\left.+\cdots+\psi_{r i}^{i} . B^{r i}\right)$ with the $\psi_{j}^{i}$ 's being the corresponding coeffic ients [55]. From the expression (6) follows:

$$
y_{t}=\frac{\theta(\mathrm{B})}{\emptyset(B)} \cdot \varepsilon_{t}+\sum_{i=1}^{k} \frac{\psi^{i}(B)}{\emptyset(B)} \cdot v_{t}^{i}
$$


Logistic $s \&$ Susta ina ble Transport

Vol. 11, No. 1, February 2020, 51-76

doi: $10.2478 /$ jlst-2020-0004

$$
y_{t}=\frac{\theta(\mathrm{B})}{\emptyset(B)} \cdot \varepsilon_{t}+\sum_{i=1}^{k} \tilde{\psi}^{i}(B) v_{t}^{i}
$$

where $\tilde{\psi}^{i}$ 's are the adequate coeffic ient polynomia ls $\frac{\psi^{i}}{\emptyset}[\underline{55}$ ]. Similar expressions as for the ARMAX model can be derived for the ARIMAX model, except that the integrated (I) part must be additionally considered (via the differencing operator, similarly as for the ARIMA model) (see $[\underline{32}, \underline{60}])$.

\section{Kalman Filtering Algorithm in the State space}

Forecasting, especia lly the long-term forec asting, is cha racterized by a high level of uncerta inty due to its high dependence on socioeconomic factors; for this reason, an error level up to $10 \%$ is acceptable [29]. In this spint, applying a Kalman filtering algorithm can significantly minimize the mean of the squared model's emror. The Ka Iman filter (KF) is a set of mathematic al equations in the state space that can provide an effic ient computational (recursive) means to estimate the state of an observed process. The Ka Iman filter is named after Rudolph E. Ka Iman, who published his famous paperin 1960 describing a recursive solution to the disc rete-data linearfiltering problem. The Ka Iman filter has been hired extensively for tracking in interactive computer graphics. It has been used for motion prediction, and it is a lso used for multi-sensor (inertial-a coustic) fusion. Moreover, this filter is very powerful in several other aspects: it supports estimations of past, present, and future states, as well as it can do so even when the precise nature of the modeled system is unknown. A Ka Iman filter is also a potent tool when it comes to controlling the noisy systems, in which the electric power systems undoubtedly can be assigned [61]. According to Gaur and his colleagues [40], the main elements that affect the electric load behavior can be classified as follows:

a. Weather. This fac tor is the most essential extreme. It comprises humid ity, wind speed, temperature, precipitation, etc. The variations in these factors straight lead to the adjustment in the habit pattems of a ppliances such as heaters, a ir conditioners, coolers, and so on.

b. Time: This factor impacts electric load at different daily periods, weekdays and weekends, holidays, and year's seasons. Here, the time-dependent electric load variation can mimor the people's lifestyle, such as their work sc hedules, leisure time, sleeping pattems, etc .

c. Economy: This factor is important in the deregulated market, reflecting the variable electricity price, while the load management policy has an important impact on the electric load growth or decline trend.

d. Random disturbances: The shutdown or start-up of the enomous loa ds such as steel mill, or wind tunnels are going to lead to the load curve impulses. The other abnomal events which are priorly known but have an unc erta in effect on the load, a lso fall in the random disturbance category.

e. Customer factors: These factors include the consumption type (commercial, residential, a gric ultural, or industrial), the size of the build ings, the number of employees, as well as the number of electric utilization.

The factors mentioned above can be injected a sinputs into the Kalman filter. Since it is extremely diffic ult to deal with the complex inputs (c., d., e.), the wea ther and time factors a re usually included in the KF only [40]. The KF is usually looked through the eyes of disc rete-time linear dynamic system. The latter has the latent state space vector $\mathbf{x}^{\mathrm{T}}(k)=\left[\begin{array}{llll}x_{1}(k) & x_{2}(k) & \ldots & x_{n}(k)\end{array}\right]$ signifying the vector of the hidden states of the appliances, as well as the observation vector $y(k)$ demonstrating the smart meter device (SMD) readings. Here, $k$ represents the discrete-time moments. The delayed estimator produces the state estimates $\mathbf{x}^{\mathrm{T}}(k \mid k-1)$ and output estimates $y(k \mid k-1)$ by applying the measurements only up to the (k-1)-th output $y(k-1)$. The $\mathrm{KF}^{\prime} \mathrm{s}$ mechanism works in a two-step process, that is, the predictor step (PS), and the corrector step (CS). In the PS, the KF estimates the current load's state on the basis of its previous state, together with its cova riance uncertainty. Once 
the new SMD's measurement is observed, the estimated state vector is updated by deploying a weighted average, where the higher weight is given to the estimate with a higher certainty. In such a way, the PS and CS steps are continuing to proceed recursively. Since the linear KF often cannot satisfy the rigor demands regarding the forecasting accuracy in the case of serious nonlinearities of the given problem, there have been developed several of its nonlinear variants. Thus, in order to explore the hidden nonlinearities of the problem, the Extended Kalman filter (EKF), as well as the Unsc ented Kalman filter (UKF), are also occasiona lly used.

\section{Grey Models (GM)}

Grey system theory (GST) was firstly introduced by Deng in 1982 [62]. This theory c an deal with the observed systems that have partially unknown parameters, while the grey models need only a limited a mount of the data to estimate the unknown system's behavior. The ma in task of the GST is to extract convincing goveming laws of the observed system on the basis of the available data, regard less of their complexity or cha otic ity [62]. The $\mathrm{GM}(1,1)$ grey model is one of the most frequently used grey models, which can produce forecasts of the future primitive data points. The $G M(1,1)$ is a time series forecasting model with differential equation (DE) having the time-dependent varying coefficients. By deploying the so-called accumulated generation (AG), it becomes possible to smooth and thus lower the intensity of the uncertainty in the system. When the DE is solved, the nstep ahead predicted value of the system can be obtained. Based on using the latter, the inverse AG (IAG) can be applied to extract the predicted values of the original data [62]. According to [63], the grey forecasting models based on the grey system theory a re extensively used in networks, since the models are capable of using random variations as the grey quantity, which is changed in a certain interval. The differential equation of the grey model is essential here since it provides the means to forecast the powerload. When the derived model is suc cessfully tested aga inst adequate reliability, stability, and accuracy, it can be deployed to forecast the future load. The grey models are suitable for all three types of load forecasting, i.e., for the short-term, medium-term and longtem forecasting [63]. One of the main a dva ntages of the GMs isthat they can be developed without considering the load distribution and the changed load's trend [5]. However, their deficiency is that they are a ppropria te only for effective solving of the problems with the prevailing exponential growth trends []ㅡ.

\section{Exponential Smoothing (ES)}

Exponential smoothing is a pragmatic forecasting approach, whereby the prediction can be ca mied out from the exponentia lly weighted a verage of the past observations $[\underline{54}, \underline{55}, 64]$. The highest weight is given to the present observation, lower weight to the immediately preceding measurement, even lower weight to the measurement before that, and so on (i.e., we are dealing with the exponential decay of the influence of past historical data) [64]. The ES models are among the most common and prevalent statistical forecasting methods because of their accuracy, simplic ity, robustness, and low cost [59]. They a re also crucial for the purpose of a load forecasting in the power systems. As emphasized by Peirong and his colleagues [51], the accuracy of the model importantly depends on smoothing coeffic ients of the EF model. This study a lso demonstra tes how to seek the best smoothing coefficients.

In general, there exist three different exponential smoothing techniques: simple, single exponential smoothing (SES) (Brown's method), double exponential smoothing (DES) (Holt's method), and triple exponential smoothing (TES) (Holt-Winters method). The SES model requires a little calculation, and it is used when the data pattem neither has a cyc lic al or seasonal va riation, nor the trend in the historical data. On the other side, the DES models, which are partic ularly used in economics, enable the forecasted values with a trend included. Fina lly, the TES (Holt-Winters) models have two possible modes of computation: the additive and the multiplic ative. The additive model is used if the original data are showing stable seasonal fluctuations. Conversely, the multiplicative models are used when the original data are reflecting the significant variations of the seasonal fluctuations [흐] . 
According to the empirical evidence, the basic Holt-Winters method tends to produce overforec asts or und er-forec a sts, partic ula rly for the longer forec a sting horizons [ 53 , 54]. For this rea son, in the year 1989, Gardnerand Mc Kenzie have deployed a new parameter $\phi$ associated with the trend component. This way, the trend is dampened to a flat line, when the future becomes more distant. Consequently, we can get the Holt-Winters Damped Additive model in the following form, including the four parameters $\alpha, \beta, \gamma, \phi[\underline{53}, \underline{54}]$ :

$$
\begin{aligned}
& \text { Level: } \quad \ell(t)=\alpha \cdot(y(t)-s(t-m))+(1-\alpha) \cdot[\ell(t-1)+\phi \cdot b(t-1)] \\
& \text { Growth: } \quad b(t)=\beta \cdot[\ell(t)-\ell(t-1)]+(1-\beta) \cdot \phi \cdot b(t-1) \\
& \text { Seasonality: } s(t)=\gamma \cdot(y(t)-l(t-1)-\phi \cdot b(t-1))+(1-\gamma) \cdot s(t-m) \\
& \text { Forecast: } \quad \hat{y}(t+h \mid t)=\ell(t)+\phi_{h} \cdot b(t)+s\left(t-m+h_{m}^{+}\right), \\
& \text {where: } \phi_{h}=\phi+\phi^{2}+\ldots+\phi^{h}, h_{m}^{+}=[(h-1) \bmod m]+1, m-\text { number of seasons |year } \\
& h \text {-time points of the future horizon } \\
& y \text {-observed time series }
\end{aligned}
$$

\section{Models of Artificial Intelligence, Computational Intelligence, or Machine Leaming}

Traditiona I sta tistic a I models a re limited and sometimes might lead to unsa tisfactory solutions. The reason is the too high number of computational possibilities leading to large solution times and the complexity of certain non-linear data pattems. Hence, machine leaming and artificial intelligencebased techniques provide a promising and attractive altemative $[15, \underline{56}]$.

\section{Artificial Neural Network (ANN) Algonithms}

The artificial neural network (ANN) approach was discovered in 1990 by Wa ren McCulloch and Walter Pitts as an altemative mechanism to the time series forecasting. The ANNs have been suc cessfully applied in many different a reas, especially for forecasting and classification purposes. ANNs models have been used and studied intensively as a tool to be used for electric load forecasting and gained huge popularity in the last few decades [56]. Basically, the neural network is a non-linear circuit that is capable of doing non-linear curve fitting. It represents an information processing paradigm that was inspired by the way the biologic al systems of humans, such as the brain, are able to process a certa in piece of information. In this process, the ANNs try to recognize regularities and pattems in the input data, leam from experience, and then provide generalized results based on their known previous knowledge. An ANN is composed of several interconnected processing elements $(\mathrm{PE})$, called neurons, which are changing their dynamic state response with respect to extemal inputs $[\underline{3}, \underline{15}, \underline{56}$. The simplest form of a $n$ a rtific ial neural network conta ining input, hidden and output layers is shown in Fig. 2. Here, the input values to the hidden node (I), associated weights (wi), hidden layer function $\mathrm{f}(\mathrm{x})$, and the output results $(\mathrm{Y})$ can be seen. By changing the weights of the ANNS, the preferred output from a specific input can be achieved. Such a process, where the weights of the ANNs are adjusted, is an iterative training process [66]. The commonly employed ANN a lgorithms for elec tric load forec asting a re $[\underline{3}, \underline{5}, \underline{14}, \underline{16-18}, \underline{39}, \underline{41}, \underline{5}, \underline{57}, \underline{66-70}]$ :

- feed-forward (F) neural networks,

- NARX (nonlinear autoregressive with exogenous inputs) neural networks,

- back-propagation (BP) neural networks,

- radial basis function (RBF) neural networks,

- the random neural networks,

- recurrent neural networks, and 


\section{- self-organizing competitive neural networks.}

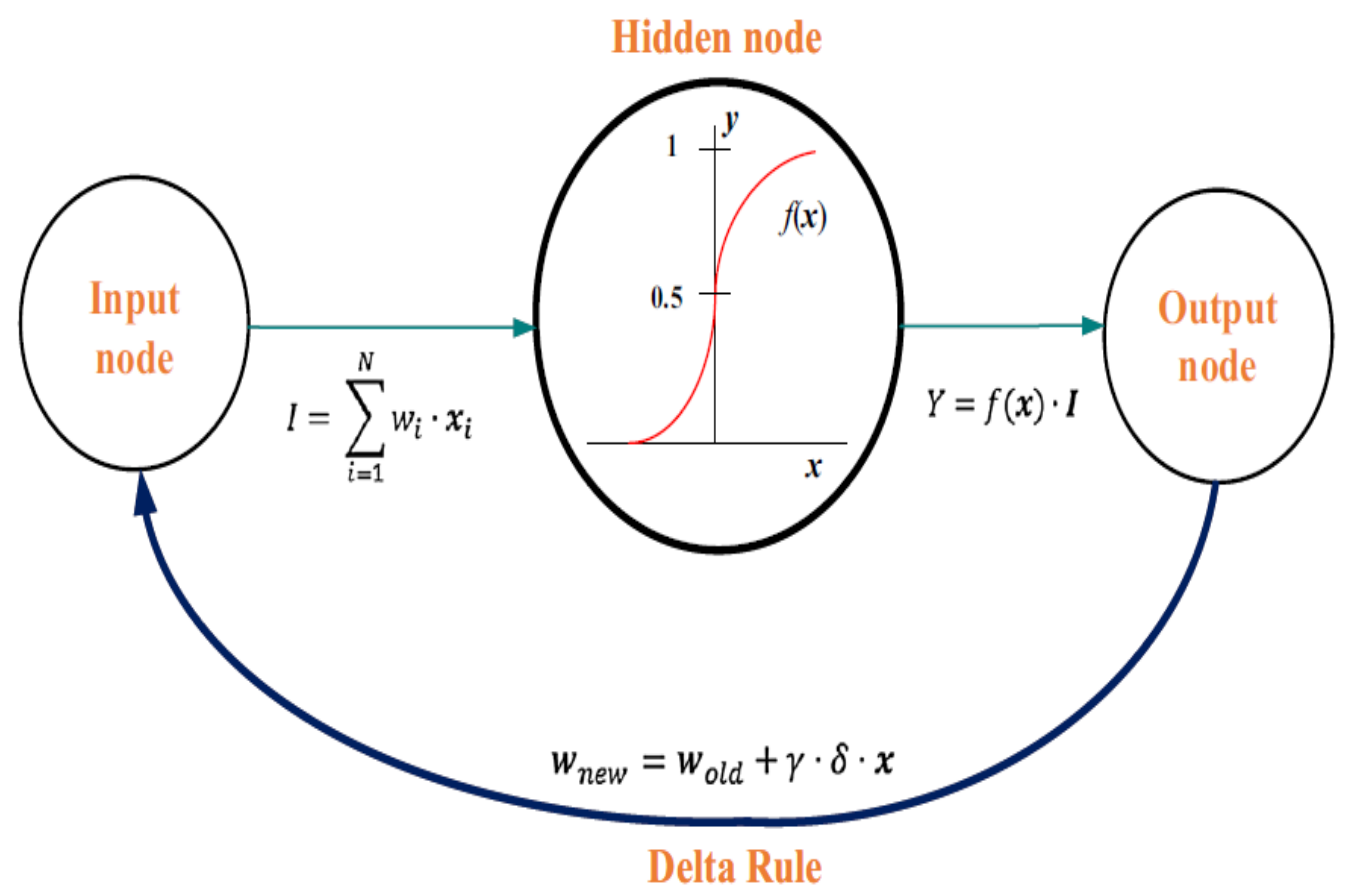

Figure 2: A simple neuron scheme in an ANN (Source: Nalc aci, Özmen, and Weber (2018)[]]).

According to [55], the proposed ANN models could be classified into the two main groups:

- The first group includes those ANNs that have only one output node, used to forecast the next hour'sload, next day'speak load, or next day's total load.

- The second group involves models that have several output nodes to forecast a sequence of hourly loads, typically 24 nodes, with a purpose to predict the next day's entire load profile.

Based on [ $\underline{5}, \underline{9}]$, the second group has strong robustness and strong lea ming ability. However, the ANN quickly falls into the local minimum because of the restriction on the generalization ability and cannot make full use of information due to the small sample size selected. Besides, the leaming convergence speed is slow.

According to (Momani et al., 2015) [66], the NARX ANNs usually outperform the classic al neural networkssuch as, for exa mple, the FF time-delay networks. Besides, the NARX networks, together with the RBF networks, are well-known to possess some excellent properties such as their simplicity, reliability, low \% error, high accuracy, and ability to create the nonlinear relationships between variables. In the aforementioned work of Momani and his colleagues, it is demonstrated that the NARX and RBF ANNs a re able to provide highly accurate EL forec asts for five days a head, while the rela tive error keeps its va lues below the rea so nable level between $0.1 \%$ to $3.9 \%$ [66]. In this study, the NARX's output $y(t)$ represents the predicted hourly load (in megawatts), which is a function of the inputs, including the humidity $u_{1}(t)$ and its past values, temperature $u_{2}(t)$, and its historical data, and the prior hourly load values $y(t-i), i=1, \ldots, n_{y}$. Accordingly, the NARX ANN model can be mathematic ally described in the following form for this case study [66]:

$$
y(t)=f_{N A R X}\left(u_{1}(t), u_{1}(t-1), \ldots, u_{1}\left(t-n_{u_{1}}\right), u_{2}(t), u_{2}(t-1), \ldots, u_{2}\left(t-n_{u_{2}}\right), y(t-1), y(t-2), \ldots, y\left(t-n_{y}\right)\right)
$$




\section{The Extreme Leaming Machines (EM)}

The Extreme leaming machines represent the special class of the FF ANNs and are appropriate for regression, classification, clustering, feature leaming, and sparse approximation. They can be used for forecasting purposes as well. Huang, Zhu, and Siew proposed the extreme leaming machines in 2004 [71]. They usually address a single-hidden layer FF neural network. In the ELM, the weights of hidden layer nodes are randomly selected, and a least-squares solution can a na lytically determine the output weights of ELM. The latter means that besides the weights that are connecting inputs to hidden nodes, the parameters of the hidden nodes need not be adjusted as well. Conversely, the hidden nodes can be randomly allocated and, afterward, never updated. The output weights of the hidden nodes are usua lly settled in a single step, which substantia lly dec reases the time needed for the leaming of the ANN. According to Huang and his colleagues [71], the ELM networks are capable of producing good generalization perfomance and can leam even thousands of times quicker than the competitive networks, which are trained using backpropagation. It is a lso detected from the literature that ELM models can outperform even the support vector machines, which are providing the sub-optimal solutions in both regression and classific ation problems.

The ELM models have also been extensively used in the field of the electric load forecasting, see for example works [72-75]. There have also been successful attempts to improve the basic ELM scheme, e.g., Ertugrul [74] has applied a novel recurrent ELM approach for the purpose of ELF forecasting, Garcia-Laencina [76] has deployed a mechanism to improve the forecasting by conducting a linearcombination of multiple ELM machines and demonstrated the performance on three engineering problems, etc. The basic scheme of the ELM network is shown in Fig. 3. [74, 77].

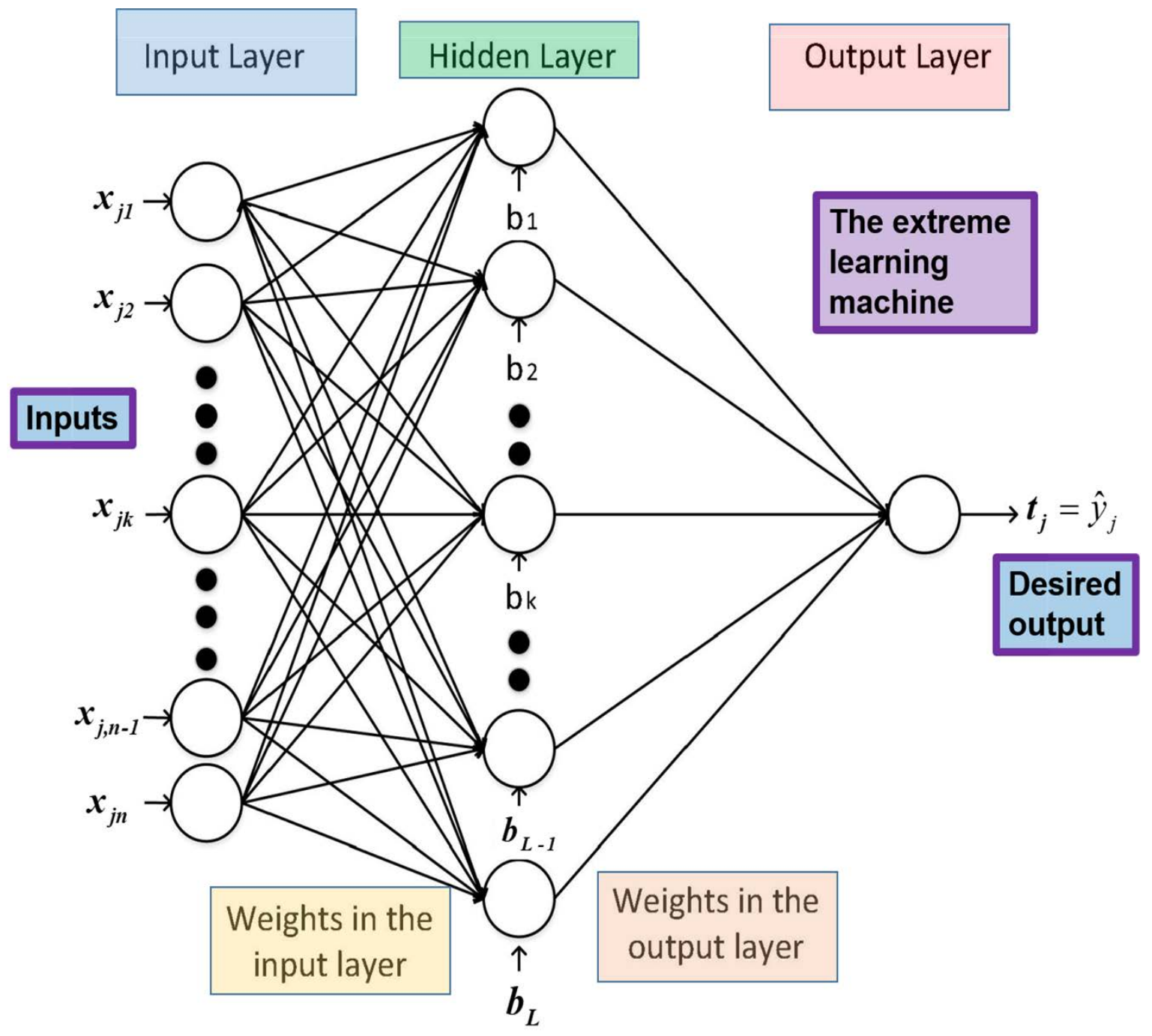

Figure 3: Diagram of the Extreme Lea ming Machine (Sources: Ertugrul; Albadr et al. [74, 17]) 
Let us assume that we have $\mathrm{N}$ arbitrary unique samples $\left(\boldsymbol{X}_{\boldsymbol{i}}, \boldsymbol{y}_{\boldsymbol{i}}\right), \boldsymbol{X}_{\boldsymbol{i}}=\left[x_{i 1}, x_{i 2}, \ldots, x_{i n}\right]^{T} \in R^{n}, \boldsymbol{y}_{\boldsymbol{i}}=\left[y_{i 1}, y_{i 2}, \ldots, y_{i m}\right]^{T} \in R^{m}$. Mathematically, the ELM can be represented by the following equation (see Fig. 3) $[71,74,77]$ :

$$
\sum_{i=1}^{L} \boldsymbol{\beta}_{\boldsymbol{i}} \cdot g_{i}\left(\boldsymbol{X}_{\boldsymbol{j}}\right)=\sum_{i=1}^{L} \boldsymbol{\beta}_{\boldsymbol{i}} \cdot g_{i}\left(\boldsymbol{W}_{i} \cdot \boldsymbol{X}_{\boldsymbol{j}}+b_{i}\right)=\sum_{i=1}^{L} \boldsymbol{\beta}_{\boldsymbol{i}} \cdot g_{i}\left(W_{i} \cdot X_{j}+b_{i}\right)
$$

Here, $g_{i}(\bullet)$ are the activation functions, $W_{i}=\left[W_{i 1}, W_{i 2}, \ldots, W_{i n}\right]^{T}$ is the vector, whose weights link the hidden node and the $i$-th input nodes, while $\boldsymbol{\beta}_{\boldsymbol{i}}=\left[\beta_{i 1}, \beta_{i 2}, \ldots, \beta_{i m}\right]^{T}$ is the vector, whose weights link the hidden node and the i-th output nodes. The parametes $b_{i}$ represent the $i$ th hidden node's threshold (bias), while the product $\boldsymbol{W}_{\boldsymbol{i}} \cdot \boldsymbol{X}_{\boldsymbol{j}}$ signifies the inner product between $\boldsymbol{W}_{\boldsymbol{i}}$ and $\boldsymbol{X}_{\boldsymbol{j}}[\underline{71}, \underline{74}, \underline{77}$. The value $L$ is the number of the neurons in the hidden layer. The lea ming a lgorithm is based on trying to obtain the zero error mean of the $\mathrm{N}$ training data, which means that the following condition must be achieved: $\sum_{j=1}^{L}\left\|\boldsymbol{y}_{j}-\boldsymbol{t}_{j}\right\|=0, t$-desired output. Then it can be shown that there exist such $\boldsymbol{\beta}_{i}, \boldsymbol{W}_{\boldsymbol{i}}, b_{i}$ that the following system of equations can be written $[\underline{71}, \underline{74}, \underline{77}]$ :

$\sum_{i=1}^{L} \boldsymbol{\beta}_{i} \cdot g_{i}\left(W_{i} \cdot X_{j}+b_{i}\right)=\boldsymbol{t}_{j}, j=1, \ldots, N$

follows:

$\boldsymbol{\beta}_{1} \cdot g_{1}\left(W_{1} \cdot X_{j}+b_{1}\right)+\boldsymbol{\beta}_{2} \cdot g_{2}\left(W_{2} \cdot X_{j}+b_{2}\right)+\ldots+\boldsymbol{\beta}_{L} \cdot g_{L}\left(W_{L} \cdot X_{j}+b_{L}\right)=\boldsymbol{t}_{j}, j=1, \ldots, N$

follows:

$\boldsymbol{\beta}_{1} \cdot g_{1}\left(W_{1} \cdot X_{1}+b_{1}\right)+\boldsymbol{\beta}_{2} \cdot g_{2}\left(W_{2} \cdot X_{1}+b_{2}\right)+\ldots+\boldsymbol{\beta}_{L} \cdot g_{L}\left(W_{L} \cdot X_{1}+b_{L}\right)=\boldsymbol{t}_{1}$

$\boldsymbol{\beta}_{1} \cdot g_{1}\left(W_{1} \cdot X_{N}+b_{1}\right)+\boldsymbol{\beta}_{2} \cdot g_{2}\left(W_{2} \cdot X_{N}+b_{2}\right)+\ldots+\boldsymbol{\beta}_{L} \cdot g_{L}\left(W_{L} \cdot X_{N}+b_{L}\right)=\boldsymbol{t}_{N}$

Thus, we have the following vector-matrix system of equations $[\underline{71}, \underline{74}, \underline{77}]$ :

$$
\underbrace{\left[\begin{array}{cccc}
g_{1}\left(W_{1} \cdot X_{1}+b_{1}\right) & g_{2}\left(W_{2} \cdot X_{1}+b_{2}\right) & \ldots & g_{L}\left(W_{L} \cdot X_{1}+b_{L}\right) \\
g_{1}\left(W_{1} \cdot X_{2}+b_{1}\right) & g_{2}\left(W_{2} \cdot X_{2}+b_{2}\right) & \ldots & g_{L}\left(W_{L} \cdot X_{2}+b_{L}\right) \\
\ldots & \ldots & \ldots & \ldots \\
g_{1}\left(W_{1} \cdot X_{N}+b_{1}\right) & g_{2}\left(W_{2} \cdot X_{N}+b_{2}\right) & \ldots & g_{L}\left(W_{L} \cdot X_{N}+b_{L}\right)
\end{array}\right]}_{\mathbf{H}_{N \times L}} \cdot \underbrace{\left[\begin{array}{c}
\boldsymbol{\beta}_{1}{ }^{T} \\
\boldsymbol{\beta}_{2}{ }^{T} \\
\ldots \\
\boldsymbol{\beta}_{L}{ }^{T}
\end{array}\right]}_{\boldsymbol{\beta}_{L \times m}}=\underbrace{\left[\begin{array}{c}
\boldsymbol{t}_{1}{ }^{T} \\
\boldsymbol{t}_{2}{ }^{T} \\
\ldots \\
\boldsymbol{t}_{L}{ }^{T}
\end{array}\right]}_{\mathbf{T}_{N \times m}}
$$

The vector $\boldsymbol{\beta}_{L \times m}$ can then be estimated by using the Moore-Penrose generalized inverse method as follows $[\underline{71}, \underline{74}, \underline{77}]$ :

$$
\begin{aligned}
& \hat{\boldsymbol{\beta}}_{L \times m}=\mathbf{H}_{N \times L}^{+} \cdot \mathbf{T}_{N \times m}, \\
& \mathbf{H}_{N \times L}^{+}-\text {The Moore-Penrose generalized inverse for } \mathbf{H}_{N \times L}
\end{aligned}
$$

Further disc ussion and detailsabout the ELM networks and theirmore advanced variantscan be found in [71-74, 76-80]. There, a more detailed explanation about effic ient using of the ELM networks for the purpose of ELF prediction can be found as well. 


\section{Support Vector Machines (SVMs)}

The support vec tor ma chines (SVMs) are regression and classific ation mechanisms [55], which was first presented by Vapnik in 1992. Later, in 1995, the soft margin classifierwasproposed by Cortesand Vapnik utilizing the statistic al lea ming theory. Initially, the SVMs were developed to deal with pattem classification problems (e.g., a face identification, optical character recognition, early medical diagnostics, the text classification, etc.). Afterward, Vapnik has extended the use of SVMs to be deployed for the regression a lgorithms as well (i.e., the support vector regression - SVR). Over the last two decades, the SVMs have received growing attention not only for pattem recognition and regression analysis but also for the forecasting purposes and solving of the time series prediction problems $[\underline{9}, \underline{15}, \underline{55}, \underline{56}]$. The ma in objective of the SVMs is to deduct a specific decision rule with a satisfactory generalization ability by choosing some specific subset of training data, called support vectors.

In the SVM models, a nonlinear mapping of the input space into a higher dimensional feature space is deployed, and afterward, an optimally separating hyperplane is extracted. Accordingly, the complexity and quality of the SVM solutions do not directly depend on the input space. When designing an SVM model, the tra ining process that is comparable to solving of a linearly constra ined quadratic programming problem isca mied out. Therefore, conversely to the other networks' training, the SVM solutions appear to be always globally optimal and unique. On the other side, the main weakness of the SVMs is that it requires an enormous amount of computations, and consequently, the time complexity of the solutions is ra dic ally increased [9].

The SVM models have also been widely used for the ELF forecasting. Fu et al. [81] have used the SVMs to predict the next day's electric ity load of public buildings, while Hong [82] has combined the SVMs with the immune algorithm to forecast the electric load. Hu and his colleagues [83] have demonstrated that the SVM forecasting model, whose parameters were adjusted by a firefly based memetic algorithm, can signific a ntly outperform the other evolutionary-based SVR models, besides some of the classical forecasting models. Furthemore, Qiang and Pu [84] have deployed the SVMs based on the particle swarm optimization to apply a short-tem load forecasting. There have also been detected many other papers in the literature that are dealing with the ELF forecasting. The ma in objective of most of the authors was to develop efficient (nature-inspired and other) a lgorithms for an appropriate setting and adjusting of the SVMs' parameters. Some papers a re also dedicated to the automatic model selection by means of SVMs. For example, Maldona do et al. [ㄷ]] have used the ideas of SVR for feature selection purposes, which resulted in the developed mechanism for a utomatic lag selection. Here, the correct identific ation of relevant lags and seasonal pattems was demonstrated, while the entire forecasting mechanism was used to effic iently predict the electric ity demand forecasting.

The details of the derivation of the SVM (and SVR) modelscan be found in the literature (e.g., see [81-89]). When a ddressing the SVM models, the structured risk minimization risk principle is considered instead of finding the minimum empirical errors [81, 83]. If we have a training dataset $\left\{\left(x_{i}, y_{i}\right)\right\} \in R^{n} \times R, x_{i}$-input, $y_{i}$-output (target), $i=1, \ldots, n$, the objective of the SVMs is to generate the decision function $y_{\text {des }}=f_{\text {des }}(x)(\langle\bullet\rangle$ is the inner product) by means of minimization of a so-called regularized risk function $\mathrm{R}$ ( $\mathrm{b}$ is the bias) $[\underline{81}, \underline{83}]$ : 
Logistic $s \&$ Susta ina ble Transport

Vol. 11, No. 1, February 2020, 51-76

doi: $10.2478 /$ jlst-2020-0004

$y_{\text {des }}=f_{\text {des }}(x)=\langle w, \phi(x)\rangle+b$,

$w$-the weight vector (controls the model's smoothness)

$\phi(x)$-thehigh-dimensional feature space

$R=R_{\text {emp }}+\frac{1}{2} \cdot\|w\|^{2}=\frac{C}{n} \cdot \sum_{i=1}^{n} L\left(y_{i}, f_{\text {des }}(x)\right)+\frac{1}{2} \cdot\|w\|^{2}$

where:

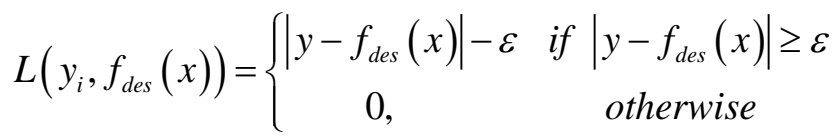

Here, $R_{e m p}$ is the empirical risk, while the function $L$ is $\varepsilon$-insensitive Vapnik's loss function, and $\varepsilon$ is named the "tube size" [됴, 83]. The second term in R, i.e., $\frac{1}{2} \cdot\|w\|^{2}$ is the so-called regularization part included as a measure of the flatness (complexity) of the function. The main purpose of the regularized constant $C$ is to find the appropriate trade-off between the regularization term and empiric al risk. The constants $C$ and $\varepsilon$ are the user-defined parameters and can significantly influence on the behavior of the entire SVM model. The equations in (10) can be transformed into the suitable form of the following minimization problem [르]:

minimize $\quad R=\frac{C}{n} \cdot \sum_{i=1}^{n} L\left(y_{i}, f_{\text {des }}(x)\right)+\frac{1}{2} \cdot\|w\|^{2}=C \cdot \sum_{i=1}^{n}\left(\xi_{i}+\xi_{i}^{*}\right)+\frac{1}{2} \cdot\|w\|^{2}$

subjected to:

$$
\begin{aligned}
& y_{i}-\left\langle w, x_{i}\right\rangle-b \leq \varepsilon+\xi_{i} \\
& \left\langle w, x_{i}\right\rangle+b-y_{i} \leq \varepsilon+\xi_{i}^{*} \\
& \xi_{i}, \xi_{i}^{*} \geq 0 \text { (slack variables), } i=1, \ldots, n
\end{aligned}
$$

Conceming the Wolfe'sdual theorem and the saddle-point condition, the dual optimization problem can also be written in the following form [요]:

$$
\underset{\alpha, \alpha^{*}}{\operatorname{maximize}}-\frac{1}{2} \sum_{i, j=1}^{l}\left(\alpha_{i}-\alpha_{i}^{*}\right) \cdot\left(\alpha_{j}-\alpha_{j}^{*}\right) \cdot\left\langle\phi\left(x_{i}\right), \phi\left(x_{j}\right)\right\rangle-\varepsilon \cdot \sum_{i=1}^{l}\left(\alpha_{i}+\alpha_{i}^{*}\right)+\sum_{i=1}^{l} y_{i} \cdot\left(\alpha_{i}-\alpha_{i}^{*}\right)
$$

subjected to:

$\sum_{i=1}^{l}\left(\alpha_{i}-\alpha_{i}^{*}\right)=0, \quad \alpha_{i}, \alpha_{i}^{*} \in[0, C] \Rightarrow$ nonnegative Lagrange multipliers

with

$w=\sum_{i=1}^{n}\left(\alpha_{i}-\alpha_{i}^{*}\right) \cdot \phi\left(x_{i}\right)$

Based on obtained $w=\sum_{i=1}^{n}\left(\alpha_{i}-\alpha_{i}^{*}\right) \cdot \phi\left(x_{i}\right)$ from (11) and the decision function $y_{\text {des }}=f_{\text {des }}(x)$ from (10), the later takes the following form [으]:

$y_{\text {des }}=f_{\text {des }}(x)=\langle w, \phi(x)\rangle+b=\left\langle\sum_{i=1}^{n}\left(\alpha_{i}-\alpha_{i}^{*}\right) \cdot \phi\left(x_{i}\right), \phi(x)\right\rangle+b=\sum_{i=1}^{n}\left(\alpha_{i}-\alpha_{i}^{*}\right) \cdot K\left(x_{i}, x_{j}\right)+b$ 
Logistic $s \&$ Susta ina ble Transport

Vol. 11, No. 1, February 2020, 51-76

doi: $10.2478 /$ jlst-2020-0004

In equation (12), $K\left(x_{i}, x_{j}\right)$ represents a kemel function. Its value corresponds to the inner product of the two vectors $x_{i}, x_{j}$ in the feature space $\phi\left(x_{i}\right), \phi\left(x_{j}\right)$. From this follows: $K\left(x_{i}, x_{j}\right)=\left\langle\phi\left(x_{i}\right), \phi\left(x_{j}\right)\right\rangle$. It tums out that any function satisfying the Mercer's condition might have been used as the kemel function (e.g., the sigmoid kemel, radial basis function kemel, polynomial kemel, etc.) [83].

\section{Fuzy Logic}

In many research fields, there have been a countless number of books (see for exa mple books [르, 90-109]) and papers a lready published about the fuzzy logic (FL), the mechanisms behind this logic, and the basic principles. Fuzzy logic is a generalization of the conventional Boolean theory, but instead of getting a value of 0 or 1 for input, it has associated with it spec ific qualitative ranges. In other words, for instance, a temperature may be low, medium, or high; however, using fuzzy logic allows outputs to be deduced from noisy or fuzzy inputs and without a need to specify a precise mapping of inputs to outputs [55]. The fuzzy methods are very useful for hand ling uncerta inties and are essential for the knowledge acquisition of human experts. A membership function can be represented for every fuzzy set, where a function for any fuzzy set, or a membership function, exhibits a specific continuous curve that is changing from 0 to 1 or vice versa, while a corresponding transition's region represents a fuzzy boundary of the term [29]. Fuzzy theory is often combined with the other methods to achieve good prediction results [5]. In short, fuzzy logic can be used in such cases [29] :

- The ma thematical model does not exist, or it exists but is too diffic ult to encode.

- The mathematical model is too complex to be evaluated fast enough for real-time operation.

- The mathematical model includes too much memory on the designated chip architecture.

- The expert is a vailable who can specify the rules underlying the system behavior and the fuzzy sets that represent the characteristic s of each variable.

- The system has unc erta inties in either its inputs or definition.

- The systemsare: too complicated, too non-linear, or with too much uncerta inty to implement using the traditional techniques.

However, we have to avoid using fuzzy logic for systems in which conventional control equations and models are already optimal or entirely adequate [29]. In the field of electric load forecasting, books $[\underline{26}, \underline{96}, \underline{102}, \underline{103}]$ are dedic ated to the modeling and prediction with a partic ula remphasis on computational and artificial intelligence approaches, including the Fuzzy logic models. Besides, Ali and his colleagues have deployed the fuzzy logic models to the short-term ELF [110], and long-term load forecasting [111]. The ELF forecasting was also addressed in works [112-115]. Further, J amaaluddin et al. [116] were dealing with a very short-term load forecasting of a peak load time by using the fuzzy logic, while J agbir and Singh [117] have conducted an FL-based short-term load forecasting model for a $220 \mathrm{kV}$ transmission line. Laouafi et al. [118] have developed an adaptive neuro-fuzzy inference system-based approach for daily load curve prediction, while Yao et al. [114] have a pplied a short-term load forec asting by interval Type-2 FL system.

\section{Wavelet Neural Networks (WNNs)}

Wavelet theory is a mathematic al theory that has been proposed by Grossman and Morlet in the 1980s. Later, Zhang has suggested the wavelet neural network (WNN) in 1992 in order to take advantage of both the wavelet functions and the widely used neural network. The WNNs are advocated asan a ltemative to the feedforwa rd neural networksfora pproximating the arbitra ry nonlinearfunctions by means of a wa velet transform theory. A WNN takes the wa velet space in the spinit of feature space for pattem recognition, and it can recognize a feature extraction of the signal by calculating the intemal product of the wavelets base and the signal vector. Hence, the network can effic iently lea $m$ the input and output cha rac teristic sof the system without too much prior info mation. 
Logistic $s \&$ Susta ina ble Transport

Vol. 11, No. 1, February 2020, 51-76

doi: $10.2478 /$ jlst-2020-0004

The signal in WNN is transmitted forward and the emor is proliferated backward, by which a more accurate predictive value of the signal is achieved. The WNNs possess rough capability and are robust for approximating the non-linear functions [14]. The comprehensive review of the ELF forecasting by means of wavelet networks can be found in Patel et al. [119].

\section{Genetic Algonithms (GA)}

In the 1950s and 1960s, there were more computer scientists, who had independently studied evolutionary systems with the idea that evolution could be used as an optimization tool for solving engineering problems. The idea in all these systems wasto develop a population of possible solutions to a given problem with the help of operators, whic $h$ mimic a genetic variation and natural selection [120]. J ohn Holland was the first who has introduced genetic algorithms and developed his idea in a book entitled "Adaptation in natural and artificial systems". David Goldberg finally popula rized the GAs in 1989. Genetic algorithms have become one of the most popular and used techniques of evolutionary computation [121]. GAs represent a plethora of optimization and search techniques based on the principle of genetic sand natural selection. They allow the population to be composed of more individuals exposed to specific selection rules that maximize the success of the solution ('fitness') or minimize the cost function. Holland presented GA as a heuristic (meta heuristic) method based on the principle of 'survival of the best'. This has made GAs a handy tool in resolving severe optimization problems [121]. To summarize, genetic algorithm represents a type of optimization algorithm to find the optimal solution(s) to a given computational problem that maximizes or minimizes a criterion function. It represents an important branch of the field of evolutionary computation [122]. In the last two decades, the GAshave been widely used and succ essfully applied to varioustypes of optimization problems [123]. The basic components of genetic algorithmsare [122, 124]:

1) A fitness function for optimization; the function that the algorithm is trying to optimize, a nd it is one of the most essential parts of the algorithm.

2) A population of chromosomes; chromosome refers to values that represent a candidate solution to the problem we are trying to solve. A genetic algorithm starts with a randomly chosen set of chromosomes, which serves as the first generation or initial population. Then each chromosome in the population is evaluated by the fitness function to test how well it solves the problem.

3) A selection of which chromosomes will reproduce; based on a probability distribution defined by the user.

4) A crossover to produce the next generation of chromosomes; the crossover operator resembles the biologic al crossing over and recombination of chromosomes in cell meiosis.

5) A random mutation of chromosomes in the new generation that randomly flips individual bits in the new chromosomes.

In the field of electric load forecasting orany kind of forecasting, the GAs have been widely used. Namely, they are frequently well-suited with nonlinear systems and they conduct a particular optimization based on the natural selection of the optimal solutions found from a wide range of the forecasting model candidates' populations [125]. Such kind of GA-based optimization is usually deployed during the model selection procedure when the most appropriate parameters of the forec asting model must be found. For instance, in work [125], the GAs have been applied to find the optimal p, d, and q parameters of the ARIMA model. Regarding the ELF forecasting, Aquino et al. [126] have employed the GAs to develop a neural-network-based load forecasting model. Gupta and Sarangi [127] have used the GA-based back-propagation method for effic ient ELF forecasting. Khan et al. [128] have reported about very short-tem load forecasting using Cartesian Genetic Programming evolved Recurrent Neural Networks. Moreover, the GA-based ELF forecasting is disc ussed in many other works, e.g., see [1229, 130]. 


\section{Expert Systems}

Based on [55], the expert system is a computer program that has the ability to explain, understand, and expand its knowledge base as new information becomes available. Expert systems combine rules and procedures used by human experts. An expert's knowledge must be convenient for codification into software rules. In particular, the experts must be able to expla in their decision process to programmers. This knowledge is later codified as facts and IF-THEN statements. It constitutes a set of relationships between the changes in the system load and changes in the exogenous factors that affect the load. Over time, some of the rules do not change, while others may have to be continuously updated.

\section{Hybrid Methods and the comprehensive classific ation of forecasting models}

Hybrid orcombination models and methodscan obtain betterforecasting performance than the single model by integrating the advantages of different single forecasting models and therefore are widely used in many forecasting areas. In this sense, there are numerous available forecasting methods, optimization algorithms, and data processing techniques for developing different hybrid models $[\underline{1}, \underline{9}]$. Thus, rec ent studies have transferred their primary resea rc $h$ foc us on the development of effective hybrid models with the hope of improving predic tion performance [14]. Accordingly, it is reasonable to find modem hybrid methods, which are presented in order to cover the new technological frameworks [131]. However, there are no confessed references on how to select different methods to build a hybrid model [9]. All the widely used electrical load forecasting models that have been aforementioned and disc ussed in the previous two sub-sections are summarized as shown in Fig. 4. 


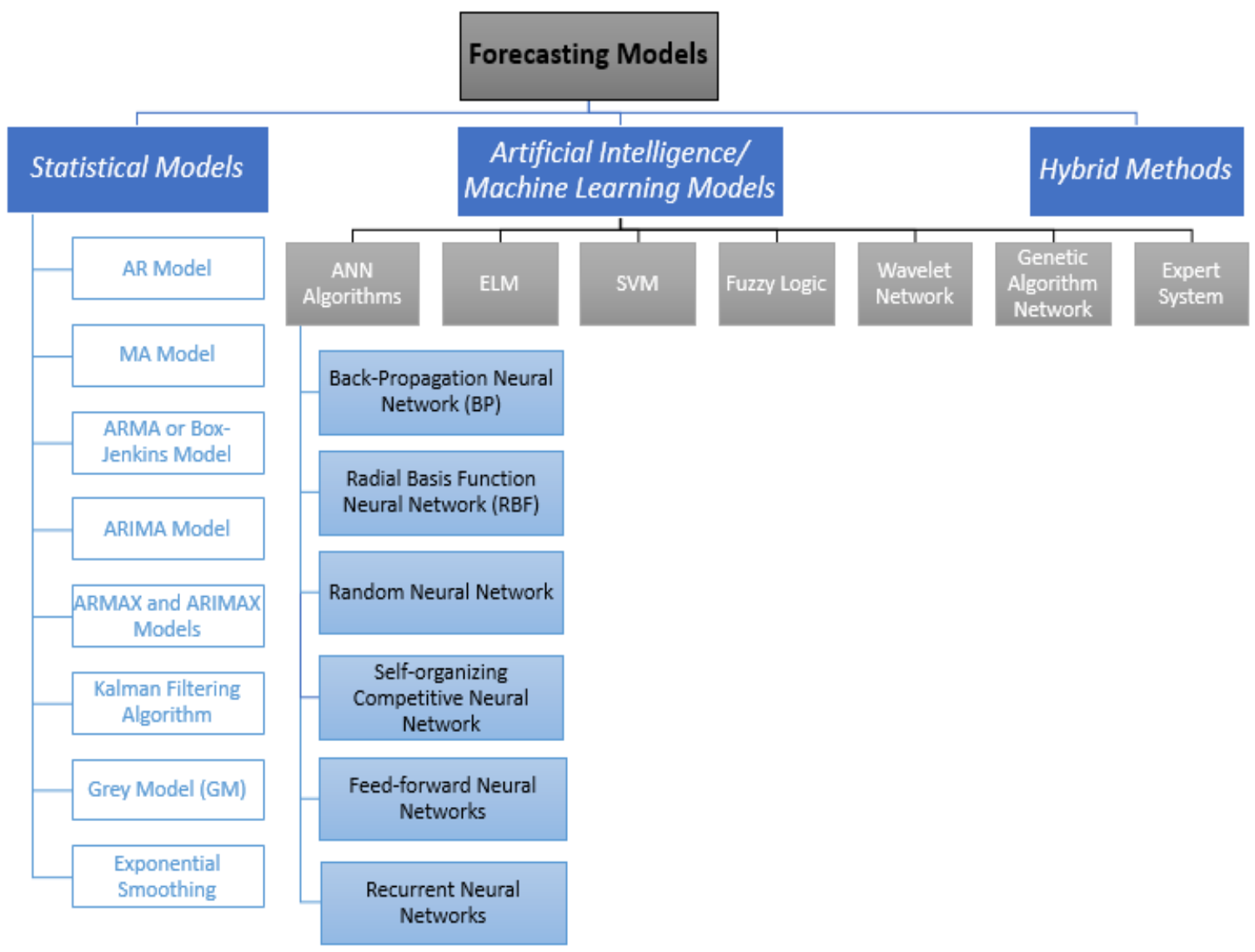

Figure 4: A comprehensive classific ation of forecasting models

Source: Adapted by the authors

\section{THE BRIEF SYSTEMIC REVIEW OF THE ELEC TRIC LOAD FO REC ASTING UTERATURE}

The study from hereafter is based on the review of academic research a imed at electricity load forecasting. Therefore, this section describes the systematic process used for the review. This study has been applied for the conventional review without the systematic review as an initial step for disc overing the general ideas of electric load forecasting models. Thus, there was no urgent need to follow the strict systematic review criteria and protocols. However, well-defined steps in order to select accurate sources and public ations were followed. Firstly, the following keywords had been used: "electric and energy", "models and methods", and "load forecasting" in English only. Searching based on these keywordshasaddressed the online database Web of Science (WOS), the most trusted citation databases in the world.

The initial search results have given $N=276$ scientific papers. Secondly, the research a rea had been narrowed down in order to find the $M=145$ most relevant studies by excluding the presence of the aforementioned keywords in the main text and leave the latter only in the context of their refeming in the title and abstract. Subsequently, a quick overview of the found works has enabled the identification of some irelevant studies and papers associated with specific keywords like: "elec tric ity pric ing", "elec tric vehic les", or "wind power". After their removal, in the end, $\mathrm{K}=52$ works have been identified and had constituted the basis of this review study. Among the selected 52 studies, 30 are artic les, 15 are conference papers (together $L=45$ papers), four a re books, and three are theses, as shown in Fig. 5. All of them have been reviewed in depth. 
Logistic $s \&$ Susta ina ble Transport

Vol. 11, No. 1, February 2020, 51-76

doi: $10.2478 /$ jlst-2020-0004

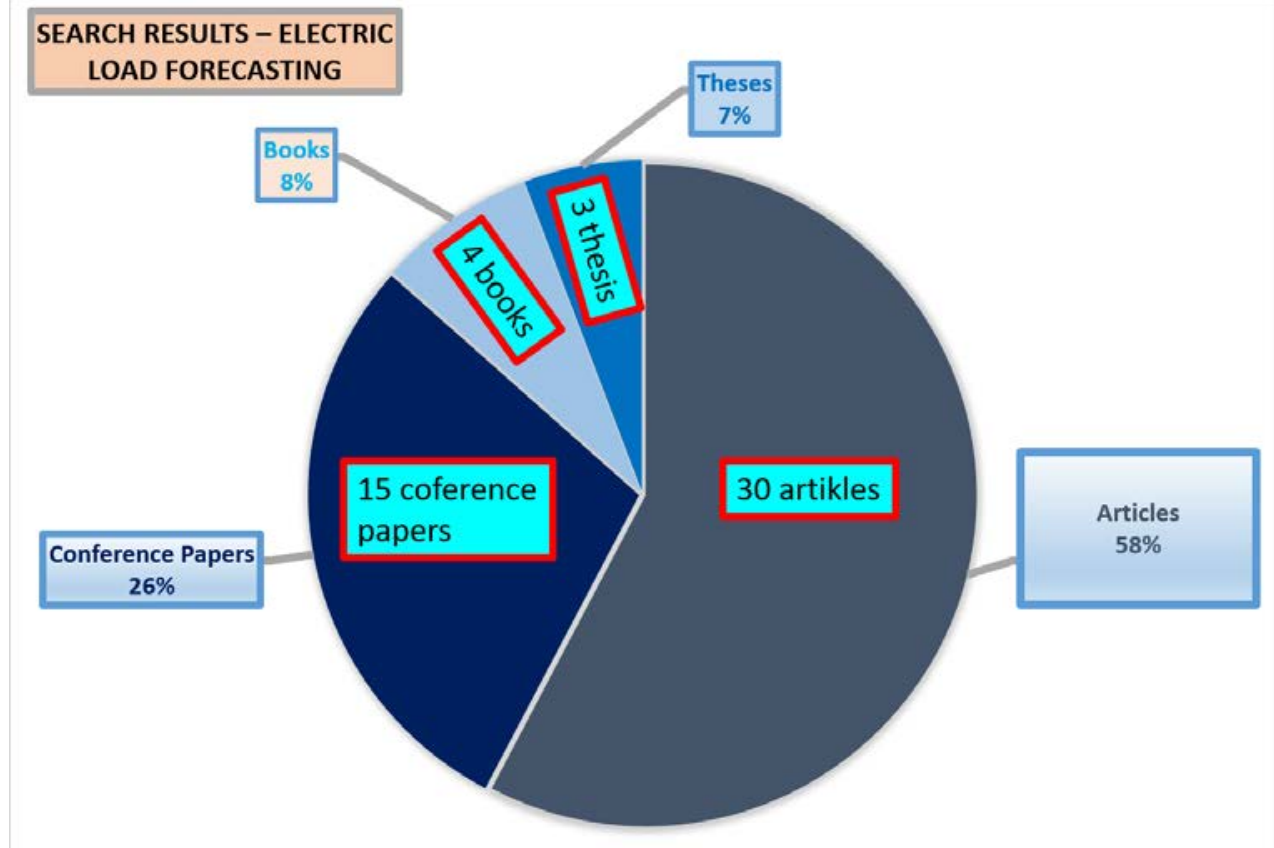

Figure 5: Search results by percentages for a review study (for $K=45$ papers)

\section{RESULTS OF THE REVIEW STUDY AND DISCUSSION}

From the $L=45$ studied papers, about 15 different models have been frequently identified. The first observation is that some models can be categorized under the same label such as: AR, MA, ARMA, ARIMA, and SARIMA models can be seen as a part of time series a nalysis (and forecasting) models. Therefore, they can be gathered into the label "Time Series models" [15]. Besides the huge number of papers presenting a load forecasting modeling in different areas of research, there are several general books and scientific theses available and published about modeling and forecasting electricity loads. Some of them only introduce the basic concepts of load forecasting modeling, while others cover all the advanced methodologies and modeling issues, let be by using statistic al or artific ial intelligence approaches. Table 1 introduces some of the typical books and theses, which dominate the field of load forecasting modeling.

Table 1: Some of available books and theses dominating at the field of load forecasting modeling

\begin{tabular}{|c|c|c|}
\hline & Author & Tite \\
\hline \multirow{5}{*}{ Books } & Rafal Weron [5 & $\begin{array}{l}\text { Modeling and Forecasting Eectricity Loads and Prices: } \\
\text { A Statistical Approach }\end{array}$ \\
\hline & $\begin{array}{l}\text { Soliman and Al- } \\
\text { Kandari [29] }\end{array}$ & $\begin{array}{l}\text { Electrical Load Forecasting: Modeling and Model } \\
\text { Construction }\end{array}$ \\
\hline & Hong et al. [20] & $\begin{array}{c}\text { Short-Term Load Forecasting by Artificial Intelligent } \\
\text { Technologies }\end{array}$ \\
\hline & $\begin{array}{l}\text { Adhikari and } \\
\text { Agrawal [ㄷ] }\end{array}$ & $\begin{array}{c}\text { An Introductory Study on Time Series Modeling and } \\
\text { Forecasting (the ELF partially covered) }\end{array}$ \\
\hline & $\begin{array}{l}\text { Hyndman and } \\
\text { Athana sopoulos } \\
\text { [30] }\end{array}$ & $\begin{array}{l}\text { Forecasting: Principles and Practice (the EF partially } \\
\text { covered) }\end{array}$ \\
\hline \multirow{3}{*}{ Theses } & Manish Singla [132] & Electrical Load Forecasting Using Neural Networks \\
\hline & $\begin{array}{l}\text { J aime Buitrago } \\
{[\underline{133}]}\end{array}$ & $\begin{array}{c}\text { Short-Tem Forecasting of Electric Loads Using Nonlinear } \\
\text { Autoregressive Artificial Neural Networks with } \\
\text { Exogenous Multivariable Inputs }\end{array}$ \\
\hline & $\begin{array}{l}\text { Mahmoud Shepero } \\
{[\underline{134}]}\end{array}$ & $\begin{array}{l}\text { Modeling And Forecasting The Load in The Future } \\
\text { Eectric ity Grid: Spatial Electric Vehicle Load Modeling } \\
\text { and Residential Load Forecasting }\end{array}$ \\
\hline
\end{tabular}


It is vital a lso to understand the general trend of researchers' interest in search and investigate the time-progress of developed models of electricity load forecasting over time in order to improve the existing results and a pplications. Thus, Fig. 6 shows the rates and number of published papers, in tems of time covera ge from 2003 to 2019 (considering L=45 papers), whereby we can notice that the trend of attention increases and the highest number of publications was recorded in 2017. The results reflect the importance of studying such a topic recently, in addition, the linear-forecast predicts a positive growing in this trend in the future.

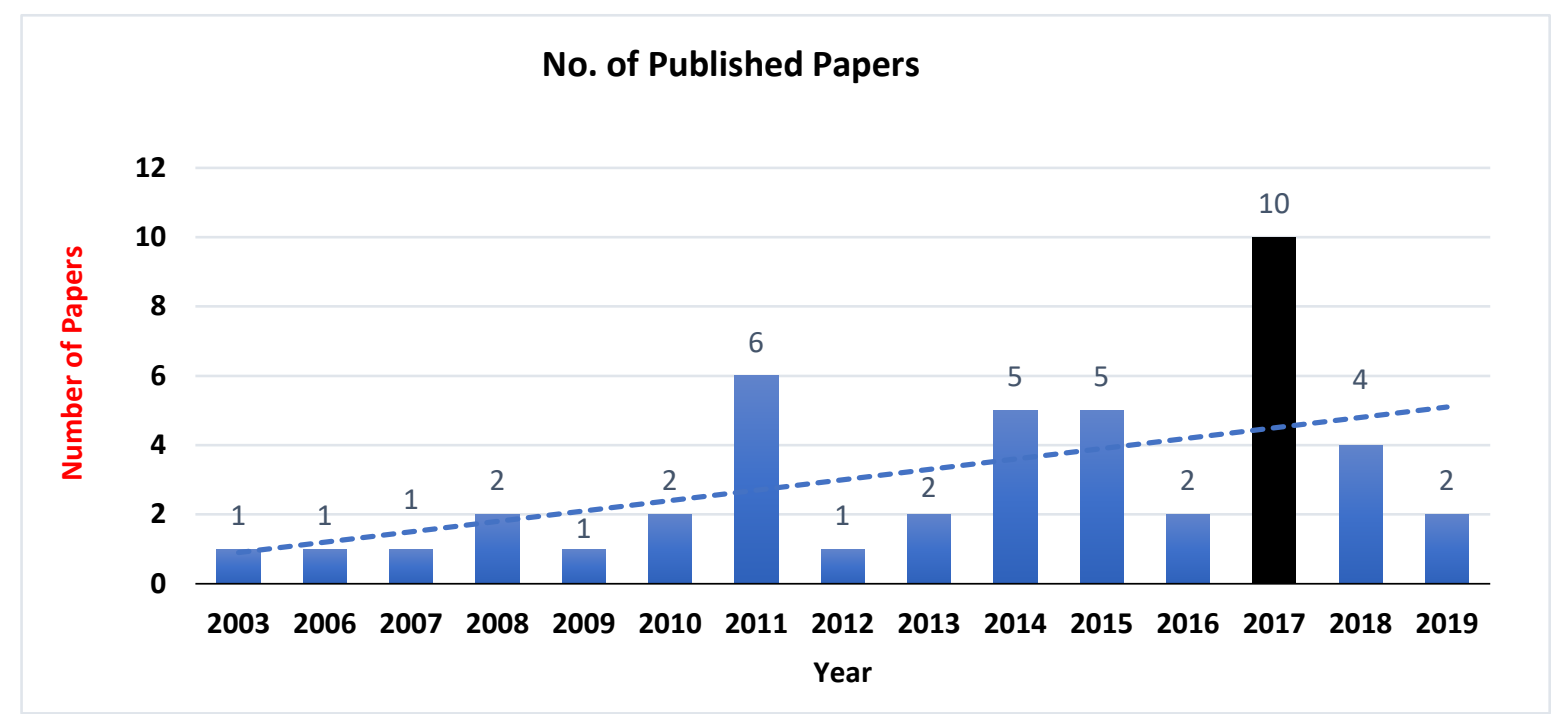

Figure 6: The Public ation Pattem in the Field of Electric Load Forecasting Modeling (for $K=45$ papers)

Fifteen different forecasting models have been identified and classified in this study. Indeed, it is interesting to study their distribution through all the $M=145$ most relevant references in order to have a clear vision for the current trend in the forecasting models used. The distribution of the different analyzed forecasting models is illustrated in Fig. 7. A clear orientation is observed in the use of forecasting models. The ANN is the most widely used present in 27 papers, followed by the regression model present in 19 papers. Then the fuzzy logic and the SVM came afterthat in 15 papers. Moreover, the ARMA and ARIMA models have been hired in 13 and 12 papers respectively, and the rest of the models were used but by limited proportions. The relatively high quantity of regression, ANN, fuzzy logic and SVM models can be explained by their popularity in the research community. This observation strengthens their status as leading models in the field. 
Logistic $s \&$ Susta ina ble Transport

Vol. 11, No. 1, February 2020, 51-76

doi: $10.2478 /$ jlst-2020-0004

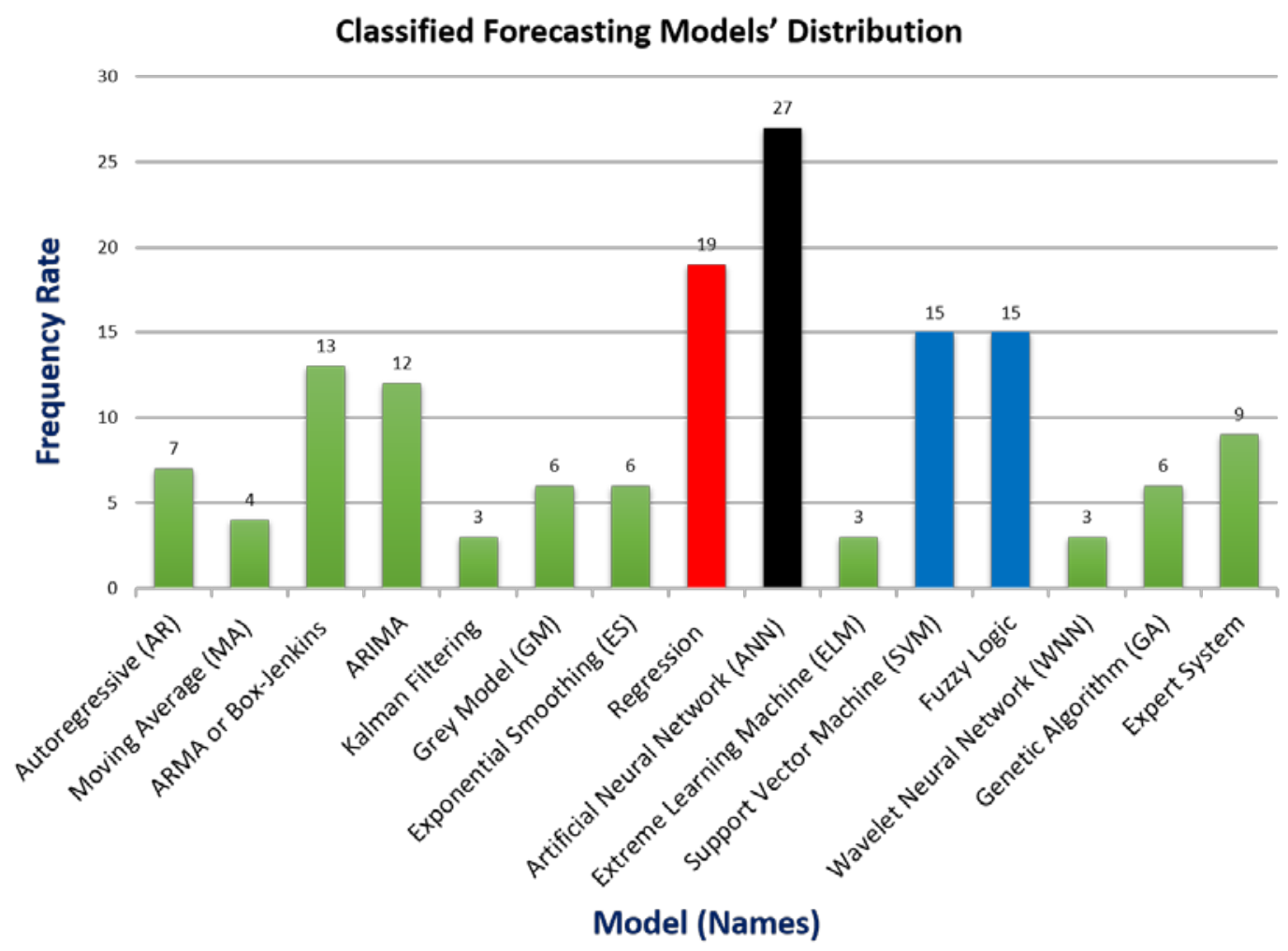

Figure 7: Distribution of Classified Forecasting Models (for $M=145$ papers)

Another important characteristic is the horizon or time-term considered for the predic tion. In fact, the time frame and solution that are chosen for any prediction will highly influence the results and the choice of a model over a nother. The timeframe of prediction is classified into four categories: very short term, short term, mid-term and long term. The forecasting horizon distribution through the different reviewed papers is shown in Table 2 . The distribution in percentage is based on the number of papers in which the forecasting time frame is relevant or emphasized. The results reveal that shortterm and long-term predictions have contributed to the highest percentage within the reviewed papers by $\mathbf{4 4 . 4 \%}$ and $\mathbf{2 2 . 2} \%$ respec tively. In contrast, very short-term and mid-term predictions are not highly represented within the cases.

Table 2: The forecasting horizon distribution through the reviewed papers (for $\mathrm{K}=45$ papers)

\begin{tabular}{c|c|c}
\hline Time Frame & $\begin{array}{c}\text { Number of Papers } \\
\text { (joumal \& Conference) }\end{array}$ & $\begin{array}{c}\text { Distribution } \\
\text { Percentage }\end{array}$ \\
\hline Very Short-Term & 1 & $2.22 \%$ \\
\hline Short-Term & $\mathbf{2 0}$ & $\mathbf{4 4 . 4 4 \%}$ \\
\hline Mid-Term & 5 & $11.11 \%$ \\
\hline Long-Term & $\mathbf{1 0}$ & $\mathbf{2 2 . 2 2} \%$ \\
\hline None & 9 & $20 \%$ \\
\hline Total & $\mathbf{4 5}$ & --- \\
\hline
\end{tabular}

In terms of geographical coverage, China is the most addressed and studied country through 4 studies followed by the USA and Turkey, then Australia and UAE, as shown in Fig. 8. 
Logistic $s \&$ Susta ina ble Transport

Vol. 11, No. 1, February 2020, 51-76

doi: $10.2478 /$ jlst-2020-0004

No. of Papers and Cases Per Country

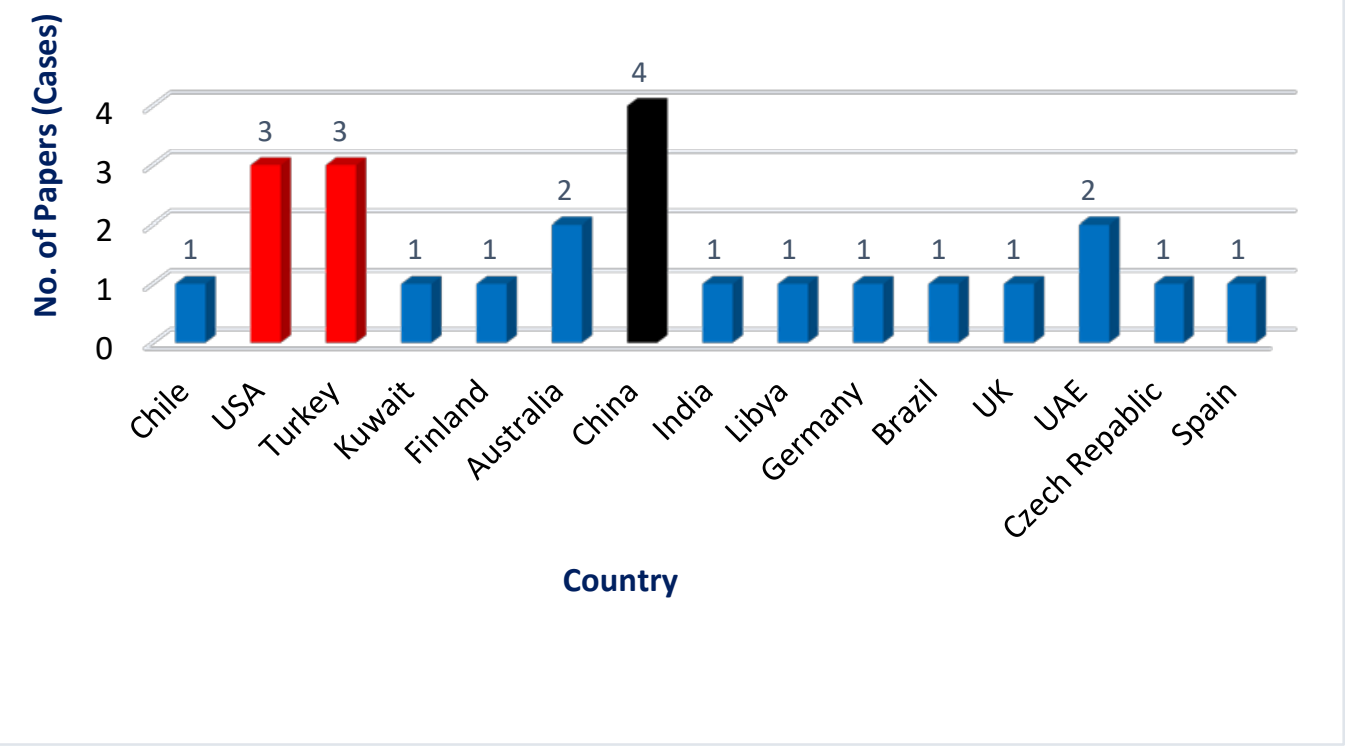

Figure 8: Coverage by Area for the Studied Countries (for $K=45$ papers)

\section{CONCLUSIONS AND FURTHER RESEARCH}

In this paper, over 15 different forecasting models distributed into 45 most relevant scientific papers about the electric load forecasting have been more in-depth investigated. Several criteria have been checked and examined such as the scale of the project, the prediction horizon time frame, time resolution, inputs, outputs, data pre-processing, etc. The study a lso a nalyzed some pattems in the use of these models. Some of them are more appropriate and preferred for electric load forecasts such as regression a nalysis based models and artificial neural networks (ANN), which are the most utilized models in electric ity predictions. In this scope, the artificial neural networks (ANN) models are ma inly employed for short-tem predictions where electric ity and power consumption pattems a re more complic ated. On the other side, the regression models are still widely applied and effic ient for long-term forecasting where periodic ity and changes are less significant. Additionally, the fuzzy logic and support vector machine (SVM) models are present in a signific ant proportion of papers showing increasing attention thereof. Conversely, the statistical models (The Box-J enkins models' fa mily in particular) are not so dominant anymore as have been in the past, but their share still cannot be neglectable. Despite all these detected studies, the research gate is still wide open to apply and adapt a lot of novel combined models for electric ity and power prediction. Moreover, certa in magnified attention to study very short-tem and mid-term load forecasting should have been additionally dedic ated to fulfilling the detected gap in the field.

\section{REFERENCES}

[1] Y. Lin, H. Luo, D. Wang, H. Guo, and K. Zhu, "An Ensemble Model Based on Machine Leaming Methods and Data Preprocessing for Short-Tem Elec tric Load Forecasting," Energies, vol. 10, no. 1186, 2017.

[2] M. D. Reddy, "Load Forecasting using Linear Regression Analysis in Time series model for RGUKT, R.K. Valley Campus HT Feeder," Intemational J oumal of Engineering Research \& Technology (IJ ERT), vol. 6, no. 5, 2017.

[3] G. Nalcaci, A. Özmen, and G. W. Weber, "Long-tem Load Forecasting: Models Based on MARS, ANN and LR methods," Central European J oumal of Operations Research (CEJ OR), Springer-Verlag GmbH Gemany, vol. 27, no. 2019, pp. 1033-1049, 2018.

[4] E. Almeshaiei and H. Soltan, "A Methodology for Electric Power Load Forecasting," Alexandria Engineening J oumal, vol. 50, no. 2011, pp. 137-144, 2011. 
[5] J. Zhang, "Research on Power Load Forecasting Based on the Improved Elman Neural Network," The Italian Association of Chemic al Engineering (AIDIC), vol. 51, no. 2016, pp. 589-594, 2016.

[6] M. Y. Khamaira, A. S. Krzma, and A. M. Alnass, "Long Term Peak Load Forecasting for the Libyan Network," in Conference for Engineering Sciences and Technology (CEST), 2018, vol. 1, pp. 185-193: AlJ R Publisher.

[7] O. Demirel, A. Kakilli, and M. Tektas, "Electric Energy Load Forecasting Using ANFIS and ARMA Methods," Joumal of the Faculty of Engineering and Architecture of Gazi University, vol. 25, no. 3, pp. 601-610, 2010.

[8] Q. Wang, Y. Wang, and L. Zhang, "Research on Post-Evaluation Model and Method of Electric Powe Demand Forecasting," presented at the Chinese Control and Decision Conference (CCDC), China, 2008.

[9] X. Zhanga, J. Wanga, and K. Zhang, "Short-Tem Elec tric Load Forecasting Based on Singular Spectrum Ana lysisa nd Support Vector Machine Optimized by Cuckoo Search Algorithm," Electric Power Systems Research, vol. 146, no. 2017, pp. 270-285, 2017.

[10] Ü. B. Filik, Ö. N. Gerek, and M. Kurban, "A Novel Modeling Approach for Hourly Forecasting of Long-Term Electric Energy Demand," Energy Conversion and Management, vol. 52, no. 2011, pp. 199-211, 2011.

[11] R. Gordillo-Orquera, L. M. Lopez-Ramos, S. Muñoz-Romero, P. Iglesias-Ca sa mubios, D. Arcos-Avilés, A. G. Marques, and J . L. Rojo-Álvarez, "Analyzing and Forecasting Electrical Load Consumption in Healthcare Buildings," Energies, vol. 11, no. 493, 2018.

[12] L. Friedrich and A. Afshari, "Short-Term Forecasting of the Abu Dhabi Electricity Load Using Multiple Weather Variables," presented at the 7th Intemational Conference on Applied Energy (ICAE), 2015.

[13] N. Abu-Shikhah and F. Elkami, "Medium-Tem Electric Load Forecasting Using Singular Value Decomposition," Energy Conversion and Management, vol. 36, no. 7, pp. 4259-4271, 2011.

[14] R. Wanga, J. Wangb, and Y. Xu, "A Novel Combined Model Based on Bybrid Optimization Algorithm for Electric al Load Forecasting," Applied Soft Computing J oumal, vol. 82, no. 2019, p. 105548, 2019.

[15] C. Kuster, Y. Rezgui, and M. Mourshed, "Electrical Load Forecasting Models: A Critical Systematic Review," in Susta inable Cities and Society, ed, 2017.

[16] H. K. Alfaresand M. Nazeenudd in, "Electric load forecasting: Literature survey and classific ation of methods," Intemational J oumal of Systems Science, vol. 33, no. 1, pp. 23-34, 2002.

[17] C. Kuster, Y. Rezgui, and M. Mourshed, "Elec trical load forecasting models: A critical systematic review," Susta ina ble Cities and Society, vol. 35, pp. 257-270, 2017, Art. no. Pii: \$2210670717305899.

[18] A. K. Singh, Ibraheem, S. Khatoon, M. Muazzam, and D. K. Chaturvedi, "Load forecasting techniques and methodologies: A review," in 2012 2nd Intemational Conference on Power, Control and Embedded Systems, 2012, pp. 1-10.

[19] S. Fallah, M. Ganjkhani, S. Shamshirband, and K.-w. Chau, "Computational Intelligence on Short-Term Load Forecasting: A Methodological Ovenview," Energies, vol. 12, no. 3, p. 393, 2019, Art. no. Pll: en12030393.

[20] W. C. Hong, M. W. Li, and G. F. Fan, Short-Term Load Forecasting by Artific ial Intelligent Technologies. MDPI AG, 2019.

[21] I. A. b. W. A. Razak, S. b. Majid, M. S. b. M. Aras, and A. b. Ahmad, "Electricity Load Forecasting Using Data Mining Technique," ed: IntechOpen, 2012.

[22] F. Su, Y. Xu, and X. Tang, "Short-and mid-term load forecasting using machine lea ming models," in 2017 China Intemational Electrical and Energy Conference (CIEEC), 2017, pp. 406-411.

[23] S. Hema Chandra, V. Harish, C. R. Kumar, and V. Naga juna, "A Review of Long Term Electrical Load Forecasting Methods," (in English), Artificial Intelligent Systems and Machine Leaming, vol. 4, no. 10, pp. 566-569, 2012.

[24] S. K. Panda, S. N. Mohanty, and A. K. Jagadev, "Long Tem Electrical Load Forecasting: An Empincal Study across Techniques and Domains," Indian J oumal of Science and Technology, vol. 10, no. 26, pp. 1-16, 2017.

[25] M. Jacob, C. Neves, and D. Vukadinović Greetham, "Short Term Load Forecasting," in FORECASTING AND ASSESSING RISK OF INDIVIDUAL ELECTRICITY PEAKS, vol. 33, M. N. C. V. G. D. J a cob, Ed. (Mathematic s of Pla net Earth, [S.I.]: SPRINGER NATURE, 2019, pp. 15-37.

[26] R. Weron, Modeling and Forecasting Electric ity Loads and Prices: A Statistical Approach. Wiley, 2007.

[27] B. Yildiz, J. I. Bilbao, and A. B. Sproul, "A review and analysis of regression and machine leaming models on commercial building electricity load forecasting," Renewable and Sustainable Energy Reviews, vol. 73, pp. 1104-1122, 2017, Art. no. Pii: s1364032117302265.

[28] E. A. Feinberg and D. Genethliou, "Load Forecasting," in Applied Mathematics for Restructured Electric Power Systems.Optimization, Control, and Computational Intelligence, J. H. Chow, F. F. Wu, and J. A. Momoh, Eds. Dordrecht: Springer-Verlag New York Inc, 2006, pp. 269-285.

[29] S. A.-h. Soliman and A. M. Al-Kandari, Electric al Load Forec asting: Modeling and Model Construction, 1st ed. ButterworthHeineman, 2010.

[30] R. J. Hyndman and G. Athana sopoulos, Forecasting: Principles and Practice, 2nd ed. OTexts: Melboume, Australia, 2018.

[31] R.M.Dawes, "Clinical versus Actuarial Prediction," Intemational Encyclopedia of the Social \& Behavioral Sciences, pp. 2048-2051, 2001. Elsevier Ltd.

[32] G. E. P. Box, G. M. J enkins, and G. C. Reinsel, Time series analysis: Forecasting and control / George E.P. Box, Gwilym M. J enkins, Gregory C. Reinsel, 4th ed. ed. (Wiley series in probability and statistics). Oxford: Wiley, 2008.

[33] P. S. P. Cowpertwait and A. V. Metcalfe, Introductory Time Series with R. Springer New York, 2009.

[34] A. J. Wood, B. F. Wollenberg, and G. B. Sheblé, Power Generation, Operation, and Control. Wiley, 2013.

[35] Casals, Jose, Garcia-Hiemaux, Alfredo, Jerez, Miguel, Sotoca, Sonia, Trindade, and A. Alexandre, "State-Space Methods forTime Series Ana lysis: Theory, Applications and Software."

[36] J. J. F. Commandeur and S. J. Koopman, An introduction to state space time series analysis (Practical econometrics). Oxford; New York: Oxford University Press, 2007, pp. xiv, 174.

[37] J. Durbin and S. J. Koopman, Time series a nalysis by state space methods, 2nd ed. ed. (Oxford statistical science series, no. 38). Oxford: Oxford University Press, 2012, pp. xxi, 346.

[38] L. Huang, Y. Yang, H. Zhao, X. Wang, and H. Zheng, "Time series modeling and filtering method of electric power load stochastic noise," Protection and Control of Modem Power Systems, vol. 2, no. 1, p. 7, 2017, Art. no. Pii: 59.

[39] S. Markoula kis, "Short-term load forecasting based on the Kalman filter and the neural-fuzzy network (ANFIS)."

[40] M. Gaurand A. Majumdar, "One-Day-Ahead Load Forecasting using nonlinear Kalman filtering algorithms," 2016. 
[41] J. Cheng, W. Xiong, and L Ai, "Electric Load Forecasting Based on Improved Grey Neural Network," in Recent advances in computer science and information engineering, vol. 124, Z Qian, Ed. (Lecture Notes in Electric al Engineering, no. 124-129) Heidelberg: Springer, 2012, pp. 651-655.

[42] C. Henui, B. Tao, and L. Yanzi, "Short-tem Power Load Forecasting Based on Gray Theory," TELKOMNIKA Indonesian J oumal of Electric al Engineering, vol. 11, no. 11, 2013.

[43] M. J in, X. Zhou, Z M. Zhang, and M. M. Tentzeris, "Short-term power load forecasting using grey correlation contest modeling," Expert Systems with Applic ations, vol. 39, no. 1, pp. 773-779, 2012, Art. no. Pii: s0957417411010347.

[44] J . A. S. Kelso, P. Érdi, K. J. Friston, H. Haken, J. Kac przyk, J. Kurths, L. E. Reic hl, P. Sc huster, F. Sc hweitzer, D. Somette, S. Liu, and Y. Lin, Grey Systems (no. 68). Berlin, Heidelberg: Springer Berlin Heidelberg, 2011, p. 401.

[45] S. Lu and Y. Lin, Grey systems: Theory and applications / Sifeng Liu and Yi Lin (Understanding complex systems). Berlin: Springer Verlag, 2010.

[46] S. Lu, Y. Yang, and J. Forrest, Grey data analysis: Methods, models and applications / Sifeng Lu, Yingjie Yang, Jeffrey Forrest (Computational risk management). Singapore: Springer, 2017.

[47] Y. Lu, Y. Teng, and H. Wang, "Load Prediction in Power System with Grey Theory and its Dia gnosis of Sta biliza tion," Electric Power Components and Systems, vol. 47, no. 6-7, pp. 619-628, 2019.

[48] J. Mi, L. Fan, X. Duan, and Y. Qiu, "Short-Tem PowerLoad Forecasting Method Ba sed on Improved Exponential Smoothing Grey Model," Mathematic al Problems in Engineering, vol. 2018, no. 1, pp. 1-11, 2018, Art. no. Pii: 3894723.

[49] T. Ozcan, T. Küçükdeniz, and F. H. Sezgin, "Comparative Ana lysis of Statistical, Machine Lea ming, and Grey Methods for Short-Tem Electricity Load Forecasting," in Nature inspired computing, vol. 1, I. R. Management Association, Ed. Hershey PA: IGI Global, 2017, pp. 1161-1183.

[50] H. Zhao and S. Guo, "An optimized grey model for annual power load forecasting," Energy, vol. 107, pp. 272-286, 2016, Art. no. Pii: s0360544216304066.

[51] P. Ji, D. Xiong, P. Wang, and J. Chen, "A Study on Exponential Smoothing Model for Load Forecasting," presented at the Asia-Pacific Power and Energy Engineening Conference (APPEEC), 2012.27-29 March 2012, Shanghai, China ; proceedings, Piscataway, NJ, 2012. Ava ilable: http://ieeexplore.ieee.org/document/6307555/

[52] D. Dragan, T. Kramberger, and M. Intihar, "A comparison of Methods for Forecasting the Container Throughput in North Adriatic Ports," presented at the IAME 2014, Norfolk, 2014.

[53] D. Dragan, A. Keshavarzsaleh, T. Kramberger, B. J ereb, and M. Rosi, "Forecasting USTourists' inflow to Slovenia by modified Holt-Winters Damped model: A case in the Tourism industry logistics and supply chains," Logistics \& Susta inable Transport, vol. 10, no. 1, pp. 11-30, 2019.

[54] R. J. Hyndman, Forecasting with exponential smoothing. Berlin; London: Springer, 2008.

[55] R. Weron, Modeling and Forecasting Electric ity Loads and Prices: A Statistical Approach. England: J ohn Wiley \& Sons Ltd, 2006.

[56] R. Adhikari and R. K. Agrawal, An Introductory Study on Time Series Modeling and Forecasting. LAP La mbert Academic Publishing, 2013.

[57] J. G.J etcheva, MostafaMajidpour, and Wei-PengChen, "Neural Network Model Ensembles for Building Level Electric ity Load Forecasts," Energy and Build ings, vol. 84, no. 2014, pp. 214-223, 2014.

[58] M. Sarhani and A. E. Afia, "Electric Load Forecasting Using Hybrid Machine Leaming Approach Incorporating Feature Selection," in Intemational Conference on Big Data Cloud and Applications, Tetuan, Morocco, 2015.

[59] X. Wang, K. Smith-Miles, and R. Hyndman, "Rule Induction for Forecasting Method Selection: Meta-Leaming the Cha racteristic s of Univa riate Time Series," Neurocomputing, vol. 72, no. 10-12, pp. 2581-2594, 2009.

[60] M. Intihar, T. Kramberger, and D. Dragan, "Conta inerThroughput Forecasting Using Dynamic Factor Analysis a nd ARIMAX Model," PROMET - Traffic \&Transportation, vol. 29, no. 5, pp. 529-542, 2017.

[61] G. Welch and G. Bishop, "An Introduction to the Kalman Filter," University of North Carolina, Chapel Hill2004, vol. TR 95041.

[62] E. Kayacan, B. Ulutas, and O. Kaynak, "Grey system theory-based models in time series predic tion," Expert Systems with Applic ations, vol. 37, no. 2, pp. 1784-1789, 2010.

[63] Y. Feng, "Study on Medium and Long Tem Power Load Forecasting Based on Combination Forecasting Model," Chemic al Engineering Transactions, vol. 51, no. 2015, pp. 859-864, 2015.

[64] E. Ostertagová and O. Ostertag, "The Simple Exponential Smoothing Model," presented at the Modelling of Mechanical and Mechatronic Systems 2011: The 4th Intemational conference, Faculty of Mechanical engineening, Technical university of Košice, 2011.

[65] A. Chusyain, R. N. S. Pelsni, and Bagio, "The Use of Exponential Smoothing Method to Predict Missing Service E-Report," presented at the Information Systems and Electrical Engineering (ICITSEE): 2nd Intemational Conferences on Information Technology, 2017.

[66] M. A. Momani, W. H. Alrousan, and A. T. Alqudah, "Short-Term Load Forecasting Based on NARX and Radial Basis Neural Networks Approaches for the J ordanian Power Grid "J ordan J oumal of Electrical Engineering, vol. 2, no. 1, pp. 8193, 2016.

[67] L. C. M. d. Andrade, M. Oleskovicz, A. Q. Santos, D. V. Coury, and R. A. S. Femandes, "Very short-term load forecasting based on NARX recurrent neural networks," in 2014 IEEE PES general meeting Piscata way, NJ : IEEE, 2014, pp. 1-5.

[68] J. Buitrago and S. Asfour, "Short-Term Forecasting of Electric Loads Using Nonlinear Autoregressive Artificial Neural Networks with Exogenous Vector Inputs," Energies, vol. 10, no. 1, p. 40, 2017, Art. no. PII: en10010040.

[69] W. X. J iatang Cheng and L Ai, "INEE 124 - Electric Load Forecasting Based on Improved Grey Neural Network."

[70] H. L, Y. Zhu, J. Hu, and Z Li, "A localized NARX Neural Network model for Short-term load forecasting based upon SelfOrganizing Mapping," in 2017 IEEE 3rd Intemational Future Energy Elec tronic S Conference and EC CE Asia (IFEEC 2017 - ECCE Asia), I. I. F. E. E. Conference, Ed. [Pisc ataway, NJ ]: IEEE, 2017, pp. 749-754.

[71] G.-B. Huang, Q.-Y. Zhu, and C.-K. Siew, "Extreme leaming machine: Theory and applications," Neurocomputing, vol. 70, no. 1, pp. 489-501, 2006.

[72] Y. Chen, M. Kloft, Y. Yang, C. Li, and L. Li, "Mixed kemel based extreme leaming machine for electric load forecasting," Neurocomputing, vol. 312, pp. 90-106, 2018. 
[73] S. K. Dash and D. Patel, "Short-term electric load forecasting using Extreme Leaming Machine - a case study of Indian power market," presented at the 2015 IEEE Power, Communication and Information Technology Conference (PCITC.15-17 October, 2015, Siksha 'O' Anusandhan University, Bhubaneswar, India : PCITC-2015 proceedings, [Piscataway, N] ], 2015. Available: http://ieeexplore.ieee.org/document/7438135/

[74] Ö. F. Ertugrul, "Forecasting electricity load by a novel recurrent extreme leaming machines approach," Intemational J oumal of Elec tric al Power \& Energy Systems, vol. 78, pp. 429-435, 2016.

[75] C. H. Weng, W. Ting, L. Xueyong, and R. Weera singhe, "Research on short-term electric loa d forec asting based on extreme lea ming machine," E3SWeb of Conferences, vol. 53, p. 02009, 2018.

[76] P. J. Garcia-Laencina, "Improving Predictions Using Linear Combination Of Multiple Extreme Leaming Machines," Information Technology And Control, vol. 42, no. 1, 2013.

[77] M. A. A. Albadrand S. Tiun, "Extreme Lea ming Machine: A Review "IntemationalJ oumal of Applied Engineening Research, vol. 12, no. 14, pp. 4610-4623, 2017.

[78] W. Ting and L. Xueyong, "Research on short-tem electric load forecasting based on extreme lea ming machine,"E3SWeb of Conferences, vol. 53, p. 02009, 2018.

[79] Y. Wei, H. Huang, B. Chen, B. Zheng, and Y. Wang, "Applic ation of Extreme Leaming Machine for Predicting Chlorophylla Concentration Inartificial Upwelling Processes," Mathematical Problems in Engineering, vol. 2019, pp. 1-11, 2019.

[80] Z Yang, T. Zhang, J. Lu, Y. Su, D. Zhang, and Y. Duan, "Extreme leaming machines for regression based on V-matrix method," Cogn Neurodyn, vol. 11, no. 5, pp. 453-465, Oct 2017.

[81] Y. Fu, Z Li, H. Zhang, and P. Xu, "Using Support VectorMachine to Predict Next Day Elec tric ity Load of Public Build ings with Sub-metering Devices," Procedia Engineering, vol. 121, pp. 1016-1022, 2015.

[82] W.-C. Hong, "Electric load forecasting by support vector model," Applied Mathematical Modelling, vol. 33, no. 5, pp. 2444-2454, 2009.

[83] Z Hu, Y. Bao, and T. Xiong, "Electricity load forecasting using support vector regression with memetic algorithms," Scientific WorldJ oumal, vol. 2013, p. 292575, 2013.

[84] S. Qiang and Y. Pu, "Short-tem power load forecasting based on support vector machine and particle swam optimization," J oumal of Algorithms \& Computational Tec hnology, vol. 13, p. 174830181879706, 2018.

[85] S. Maldonado, A. González, and S. Crone, "Automatic time series analysis for electric load forecasting via support vector regression," Applied Soft Computing, vol. 83, p. 105616, 2019.

[86] N. Cristianini and J. Shawe-Taylor, An introduction to Support Vector Machines. New York: Cambridge University Press, 2000.

[87] N. Deng, Y. Tian, and C. Zhang, Support vectormachines (Chapman \& Hall/CRC data mining and knowledge discovery series). Boca Raton: CRC Press, 2013.

[88] I. Steinwart and A. Christmann, Support vector mac hines (Information science and statistics). New York: Springer, 2008.

[89] J. A. K. Suykens, Least squares support vector machines. New J ersey; London: World Scientific H1 - British Library H2 - DSC $\mathrm{m03} / 18809,2002$.

[90] A. Abraham and S. Das, Computational Intelligence in Power Engineening. Springer Berlin Heidelberg, 2010.

[91] P. L. Anderson, Business Economics and Finance with MATLAB, GIS, and Simulation Models. CRC Press, 2004.

[92] M. F. Azeem, Fuzzy Inference System: Theory and Applications. IntechOpen, 2012.

[93] J. H. Chow, F. F. Wu, and J. A. Momoh, Applied Mathematics for Restructured Electric Power Systems: Optimization, Control, and Computational Intelligence. Springer US, 2004.

[94] S. K. Halgamuge and L. Wang, Computational Intelligence for Modelling and Prediction. Springer Berlin Heidelberg, 2005.

[95] R. Jensen and Q. Shen, Computational Intelligence and Feature Selection: Rough and Fuzzy Approaches. Wiley, 2008.

[96] S. Kalogirou, Artific ial Intelligence in Energy and Renewa ble Energy Systems. Nova Science Publishers, 2007.

[97] A. Konar and D. Bhattacharya, Time-Series Prediction and Applications: A Machine Intelligence Approach. Springer Intemational Publishing, 2017.

[98] E. Ogliari and S. Leva, Computational Intelligence in Photovoltaic Systems. Mdpi AG, 2019.

[99] A. K. Palit and D. Popovic, Computational Intelligence in Time Series Forecasting: Theory and Engineening Applications. Springer London, 2006.

[100] M. Paulescu, E. Paulescu, P. Gravila, and V. Badescu, Weather Modeling and Forecasting of PV Systems Operation. Springer London, 2012.

[101] W. Pedryczand S. M. Chen, Time Series Analysis, Modeling and Applications: A Computational Intelligence Perspective. Springer Berlin Heidelberg, 2012.

[102] S. A. Soliman and A. M. Al-Kandari, Electrical Load Forecasting: Modeling and Model Construction. Elsevier Science, 2010.

[103] Y. H. Song, Modem Optimisation Techniques in Power Systems. Springer Netherlands, 1999.

[104] M. Sudha, Applied Computational Intelligence. Educreation Publishing, 2019.

[105] G. Tayfur, Soft Computing in Water Resources Engineering: Artificial Neural Networks, Fuzzy Logic and Genetic Algorithms. WITPress, 2014.

[106] K. E. Voges and N. Pope, Business Applic ations and Computational Intelligence. Idea Group Publishing, 2006.

[107] J. Wang, Business Intelligence in Economic Forecasting: Technologies and Techniques: Technologies and Techniques. Information Science Reference, 2010.

[108] L. Wang, C. Singh, and A. Kusiak, Wind Power Systems: Applications of Computational Intelligence. Springer Berlin Heidelberg, 2010.

[109] P. P. Wang, Computational Intelligence in Economic s and Finance. Springer Berlin Heidelberg, 2013.

[110] A. T. Ali, E. B. Tayeb, and Z M. Shamseldin, "Short tem electrical load forecasting using fuzzy logic," Intemational] oumal Of Advancement In Engineering Technology, Management and Applied Science (IJ AETMAS), vol. 3, 2016.

[111] D. Ali, M. Yohanna, M. I. Puwu, and B. M. Garkida, "Long-tem load forecast modelling using a fuzzy logic approach," Pacific Science Review A: Natural Science and Engineering, vol. 18, no. 2, pp. 123-127, 2016.

[112] M. Faysal, M. J . Islam, M. M. Murad, M. I. Islam, and M. R. Amin, "Electrical Load Forecasting Using Fuzzy System," Joumal of Computer and Communications, vol. 07, no. 09, pp. 27-37, 2019. 


\section{Logistic $s$ \& Susta ina ble Transport \\ Vol. 11, No. 1, February 2020, 51-76 doi: 10.2478/jlst-2020-0004}

[113] M. K. Singla and S. Hans, "Load Forecasting using Fuzzy Logic Tool Box," Global Research and Development J oumal for Engineering, vol. 38, pp. 12-19, 2018.

[114] L Yao, Y.-I. J iang, and J. Xiao, "Short-Tem Power Load Forecasting by Interval Type-2 Fuzzy Logic System," in Information Computing and Applications, Berlin, Heidelberg, 2011, pp. 575-582: Springer Berlin Heidelberg.

[115] Z Ismail and R. Mansor, "Fuzzy Logic Approach for Forecasting Half-hourly Electricity Load Demand," Fuzzy Logic Approach for Forecasting Half-hourly Electricity Load Demand, 09/14 2011.

[116] J. Jamaaluddin, D. Hadidjaja, I. Sulistiyowati, E. A. Suprayitno, I. Anshory, and S. Syahrorini, "Very short term load forecasting peak load time using fuzzy logic," IOP Conference Series: Materials Science and Engineering, vol. 403, p. 012070, 2018.

[117] J. Kaur and Y. S. Brar, "Short term load forecasting using fuzzy logic of 220KV transmission line," Int. J. Eng. Res. Technol, vol. 3, no. 2278, p. e0181, 2014.

[118] A. Laouafi, M. Mordja oui, and T. E. Boukelia, "An a daptive neuro-fuzzy inference system-based approach for daily load curve prediction,"J oumal of Energy Systems, pp. 115-126, 2018.

[119] S. K. Patel and S. Shama, "A Review of very Short-Term Load Forecasting (STL) using Wavelet Neural Networks," Intemational J oumal of Sc ience, Engineering and Technology Research vol. 4, no. 2, 2015.

[120] M. Mitchell, An introduction to genetic algorithms, 7. print ed. (A Bradford book). Cambridge, Mass., 2001, p. 209.

[121] R. L. Haupt and S. E. Haupt, Practical genetic algorithms, 2nd ed. ed. Hoboken, N.J .; Chichester: Wiley-Interscience, 2004.

[122] J. Carr, "An Introduction to Genetic Algorithms," 2014.

[123] C.-C. Hsu, C.-H. Wu, S.-C. Chen, and K.-L. Peng, "Dynamic ally Optimizing Parameters in Support Vector Regression: An Application of Electric ity Load Forecasting," presented at the System Sciences, the 39th Annual Hawaii Intemational Conference, 2006.

[124] D. Bea sley, D. R. Bull, and R. R. Martin, "An Overview of Genetic Algorithms: Part 1, Fundamentals," University Computing, vol. 15, no. 2, pp. 56-69, 1993.

[125] Y. K. Al-Doun, H. Al-Chalabi, and J. Lundberg, "Time Series Forecasting using Genetic Algorithm," in The Twelfth Intemational Conference on Advanced Engineering Computing and Applications in Sciences, 2018.

[126] R. R. B. de Aquino, O. N. Neto, M. M. S. Lira, A. A. Ferreira, and K. F. Santos, "Using Genetic Algorithm to Develop a NeuralNetwork-Based Load Forecasting," in Artificial Neural Networks - ICANN 2007, Berlin, Heidelberg, 2007, pp. 738-747: Springer Berlin Heidelberg.

[127] A. Gupta and P. K. Sarangi, "Electrical load forecasting using genetic algorithm based back-propagation method," ARPN J oumal of Engineering and Applied Sciences, vol. 7, no. 8, pp. 1017-1020, 2012.

[128] G. M. Khan, F. Zafari, and S. A. Mahmud, "Very Short Tem Load Forecasting Using Cartesian Genetic Programming Evolved Recurrent Neural Networks (CGPRNN)," in 2013 12th Intemational Conference on Machine Leaming and Applications, 2013, vol. 2, pp. 152-155.

[129] F. L and X. Zhao, "The application of genetic algorithm in power short-term load forecasting," in 2012 Intemational Conference on Image, Vision and Computing (ICIVC 2012), 2012.

[130] J . I. Silva-Ortegaa, B. Cenvantes-Bolivarb, I. A. Isaa c-Millanc, Y. Cardenas-Escorcia b, and G. Valencia-Ochoad, "Demand energy forecasting using genetic algorithm to guarantee safety on electrical transportation system," CHEMICAL ENG INEERING, vol. 67, 2018.

[131] H. Verdejo, A. Awerkin, C. Becker, and G. Olguin, "Statistic Linear Parametric Techniques for Residential Electric Energy Demand Forecasting: A Review and An Implementation to Chile," Renewable and Susta inable Energy Reviews, vol. 74, no. 2017, pp. 512-521, 2017.

[132] M. Singla, "Load Forecasting Using Artificial Neural Network," Tha par Institute, 2018.

[133] J. Buitrago, "Short-Tem Forecasting of Electric Loads Using Nonlinear Autoregressive Artificial Neural Networks with Exogenous Multivariable Inputs," Open Access Dissertations, 2017.

[134] M. Shepero, "Modeling and forecasting the load in the future electricity grid : Spatial electric vehicle load modeling and residential load forec asting," ed: Uppsala universitet, 2018.

AUTHORS

A. Mahmoud A. Hammad, M.Sc., is Assistant Lecturer at the Arab Academy for Science, Technology and Maritime Transport, Alexandria, Egypt.

B. BonutJ ereb, Ph.D., is Full Professora nd the Vic e-Dean at the Fa culty of Log istics, University of Ma ribor, Celje, Slovenia (e-mail: borut.jereb@um.si).

C. Bojan Rosi, Ph.D., is Full Professor and the Dean at the Faculty of Logistics, University of Maribor, Celje, Slovenia (e-mail: bojan.rosi@um.si).

D. Dejan Dragan, Ph.D., is an Assoc iate Professor at the Fac ulty of Logistic s, University of Maribor, Celje, Slovenia (e-mail: dejan.dragan@um.si).

Manuscript received by 23 December 2019.

Published as submitted by the author(s). 